\title{
Legume Abundance Along Successional And Rainfall Gradients In Neotropical Forests
}

This PDF file includes:

Supplementary Fig. 1 to 7

Supplementary Tables 1 to 6

References (63-85) 


\section{Legume Abundance Along Successional And Rainfall Gradients In Neotropical Forests}




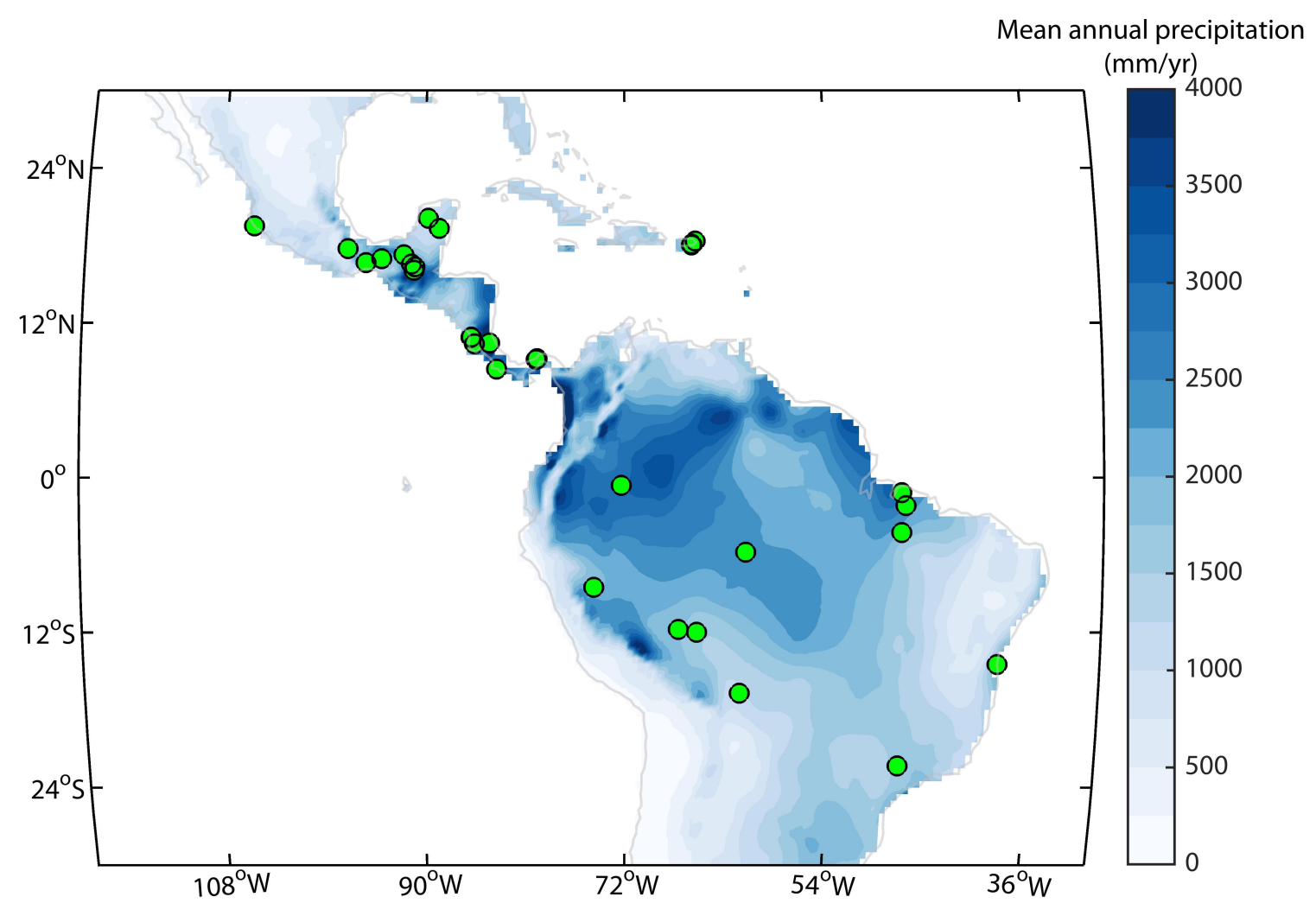

Supplementary Fig. 1 | The 2ndFOR study sites included in this study $(N=42)$. Shading illustrates mean annual precipitation between 1971 and 2010 (derived from the TS2p1 dataset, Climate Research Unit, University of East Anglia) ${ }^{63}$. 
a $\mathrm{DBH}<10 \mathrm{~cm}$

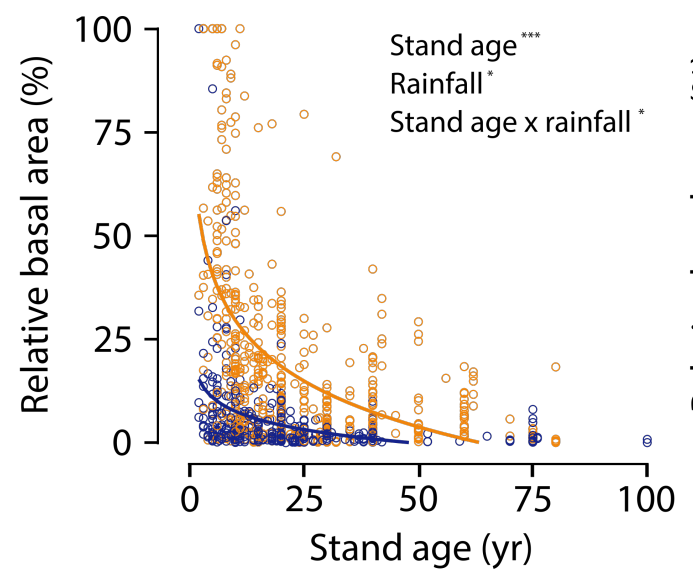

c $\mathrm{DBH} 20-30 \mathrm{~cm}$

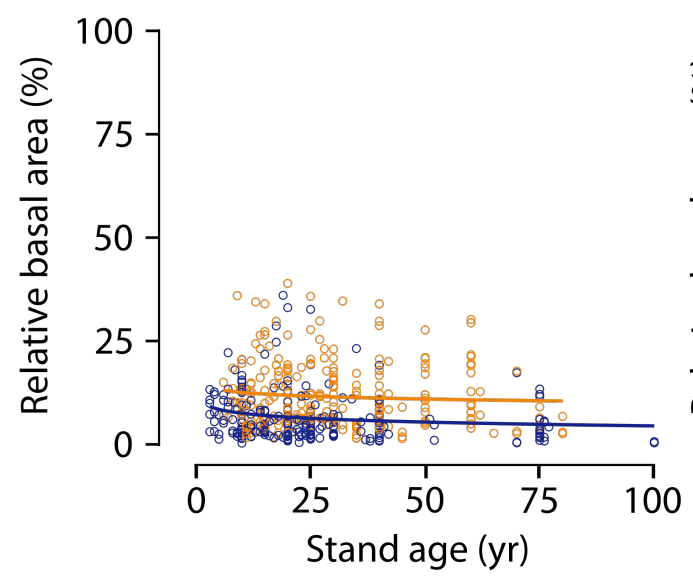

b $\mathrm{DBH} 10-20 \mathrm{~cm}$

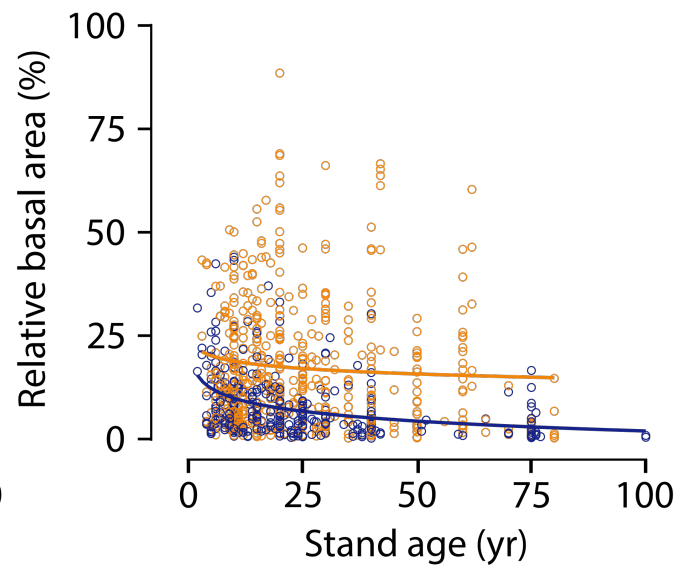

d $\mathrm{DBH}>30 \mathrm{~cm}$

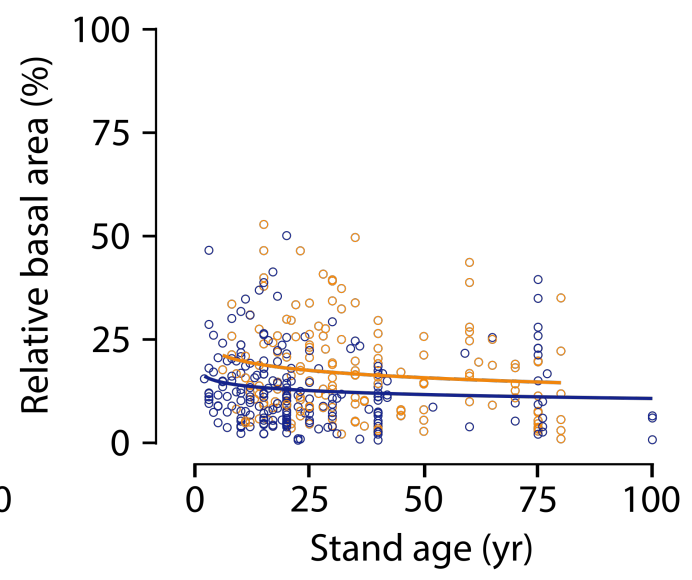

Supplementary Fig. 2 | Plot-level relative basal area of legumes in secondary dry (orange circles) and wet forests (blue circles), stratified by size classes, $N=42$ sites. Results of a linear mixed model, with stand age and rainfall (and their interaction) as fixed effects and site as random effect are shown $\left({ }^{\dagger} P<0.05 ;{ }^{*} P<0.01 ;{ }^{* *} P<0.001\right.$; ${ }^{* * *}$ $P<0.0001)$. 

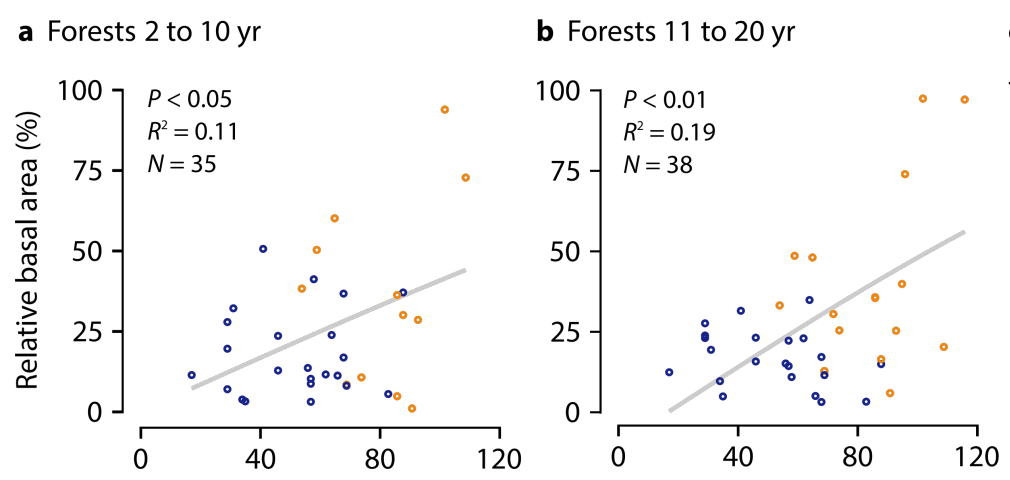

c Forests 21 to $30 \mathrm{yr}$
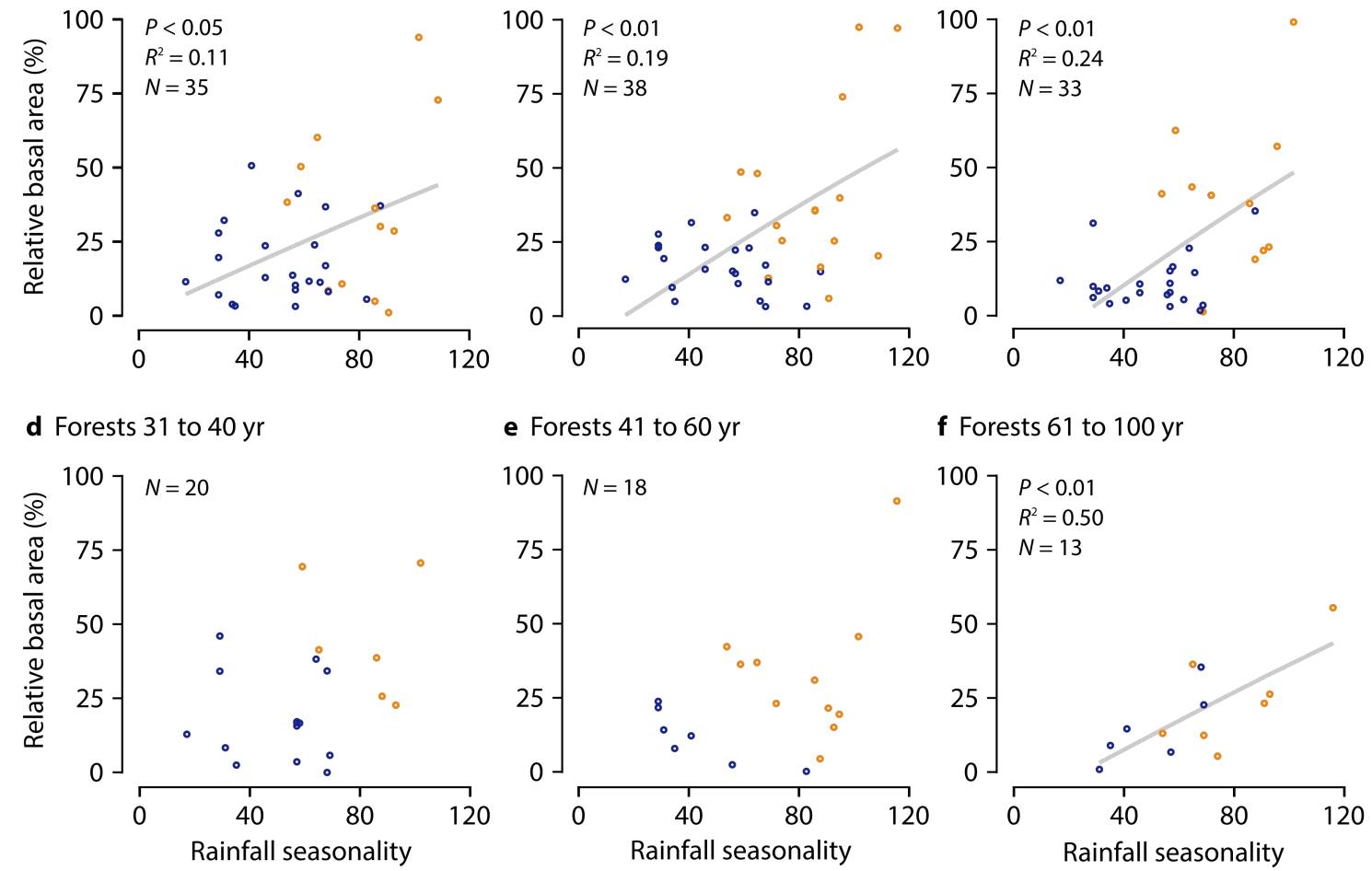

Supplementary Fig. 3 | Relationship between legume relative basal area and rainfall seasonality at sites of similar stand age.

(a) 2 to $10 \mathrm{yr}$ old forests, (b) 11 to $20 \mathrm{yr}$ old forests, (c) 21 to $30 \mathrm{yr}$ old forests, (d) 31 to $40 \mathrm{yr}$ old forests, (e) 41 to $60 \mathrm{yr}$ old forests, and (f) 61 to $100 \mathrm{yr}$ old forests. Dry forest sites are shown orange circles, and wet forests as blue circles. Results of linear regressions performed on arcsin transformed RA are shown in each panel $\left({ }^{\dagger} P<0.05 ;{ }^{*} P\right.$ $\left.<0.01 ;{ }^{* *} P<0.001 ;{ }^{* * *} P<0.0001\right)$. 

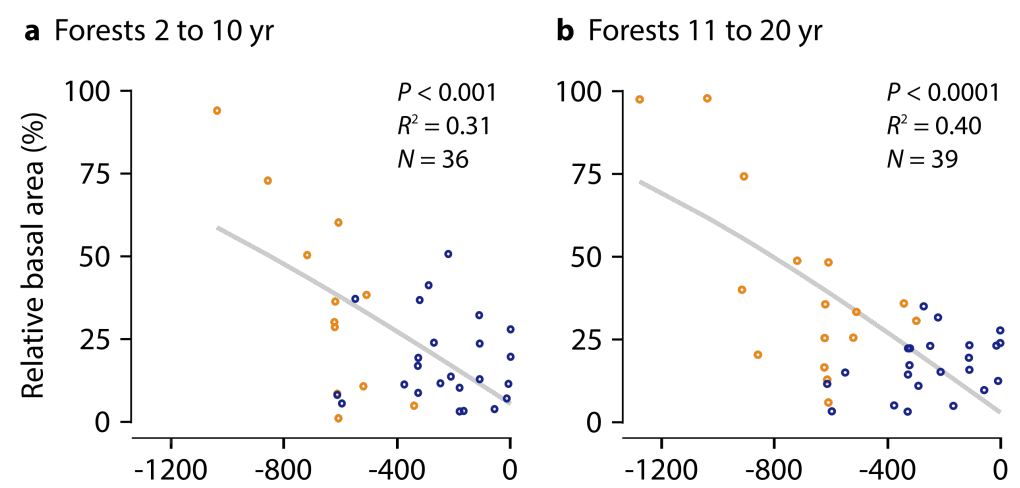

c Forests 21 to $30 \mathrm{yr}$
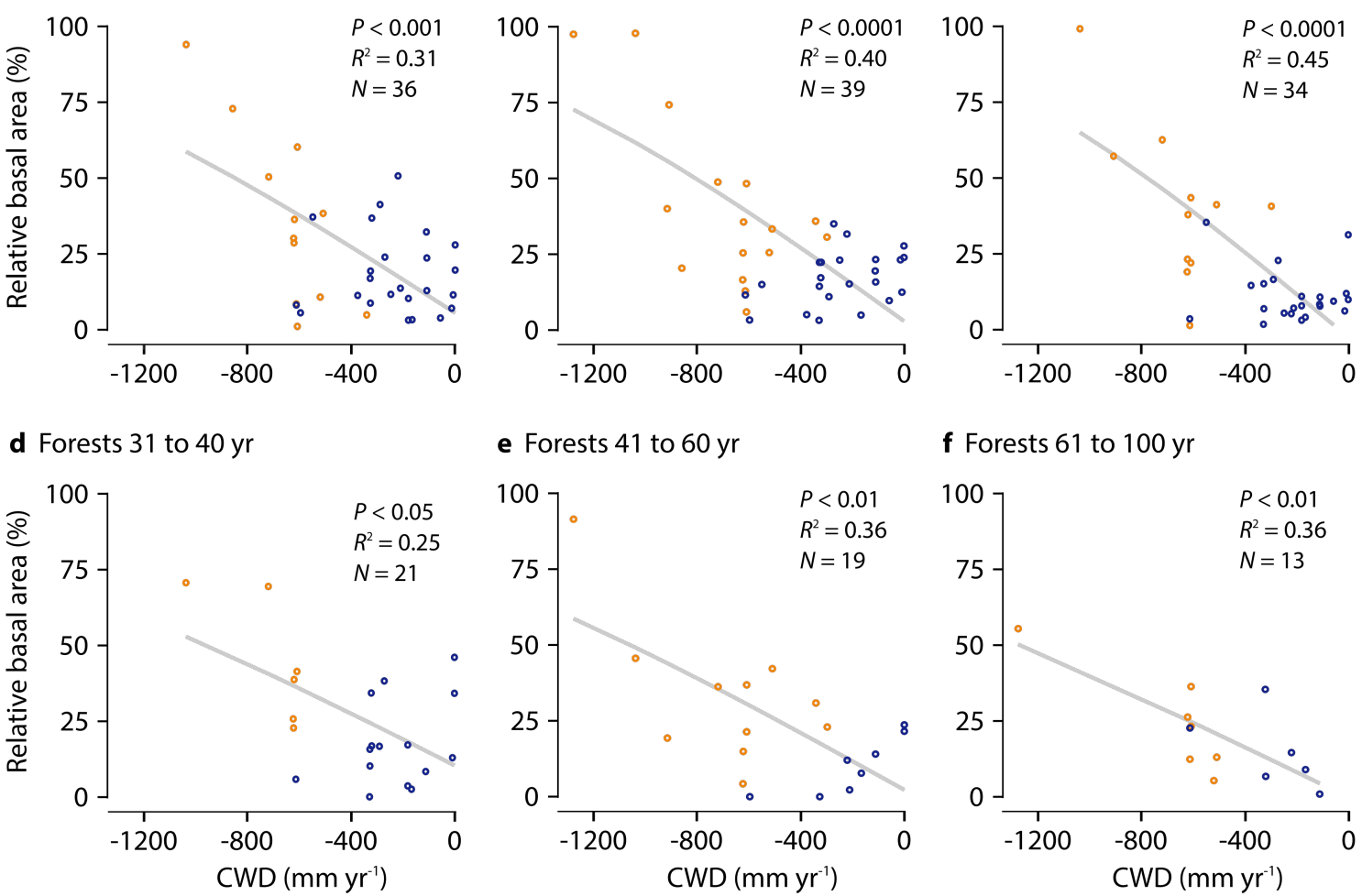

f Forests 61 to $100 \mathrm{yr}$

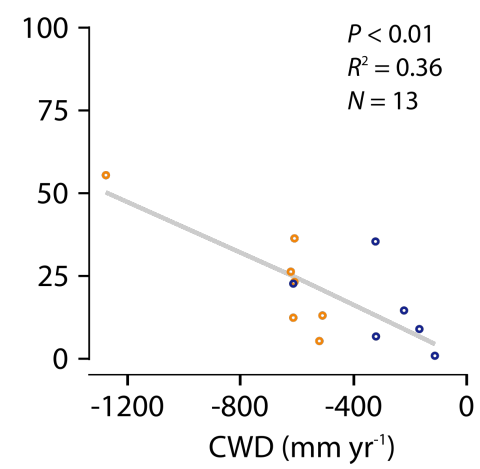

Supplementary Fig. 4 | Relationship between legume relative basal area and climatic water deficit at sites of similar stand age.

(a) 2 to $10 \mathrm{yr}$ old forests, (b) 11 to $20 \mathrm{yr}$ old forests, (c) 21 to $30 \mathrm{yr}$ old forests, (d) 31 to $40 \mathrm{yr}$ old forests, (e) 41 to $60 \mathrm{yr}$ old forests, and (f) 61 to $100 \mathrm{yr}$ old forests. Dry forest sites are shown orange circles, and wet forests as blue circles. Results of linear regressions performed on arcsin transformed RA are shown in each panel $\left({ }^{\dagger} P<0.05 ;{ }^{*} P\right.$ $\left.<0.01 ;{ }^{* *} P<0.001 ;{ }^{* * *} P<0.0001\right)$. 
$\longrightarrow$ dry forest sites $\quad \longrightarrow$ wet forest sites
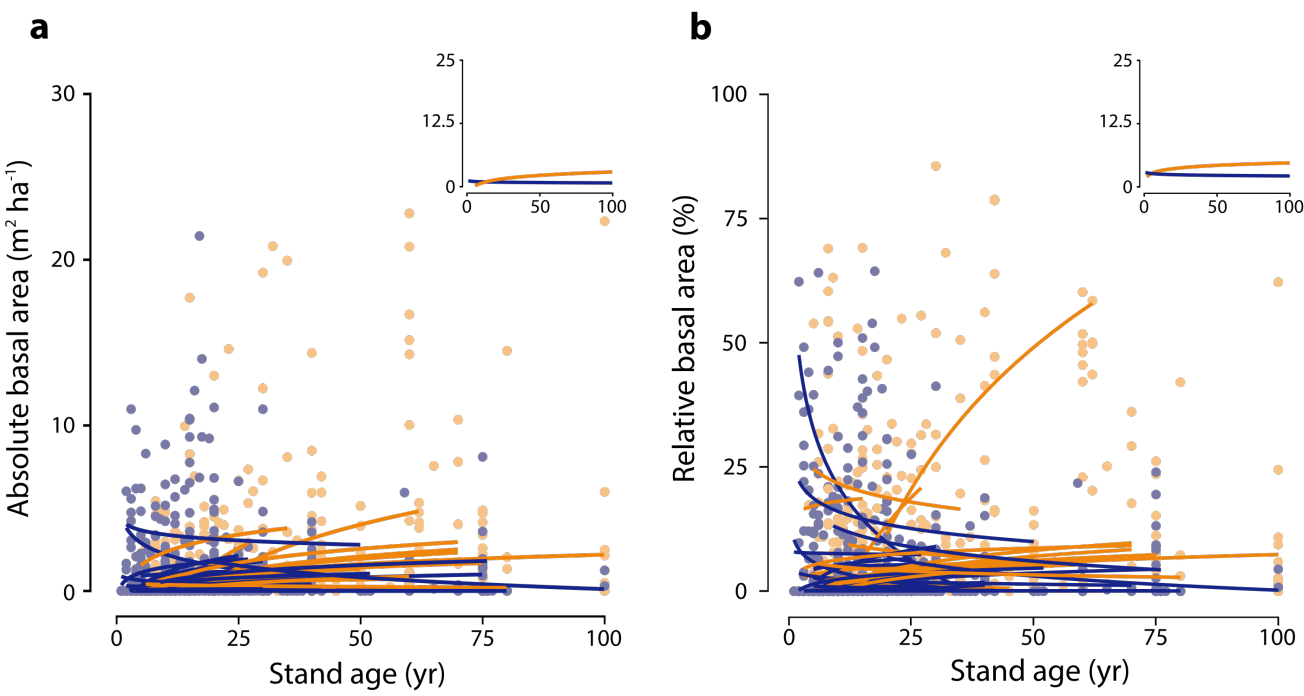

Supplementary Fig. 5 | Relationship between relative basal area of non-fixing legume species and stand age in Neotropical secondary forests, $N=42$ sites.

(a) Plot-level total basal area, and (b) relative basal area of non-fixing legumes. Each circle represents one plot and each line the successional trajectory of an individual chronosequence $(N=42)$. In panel $(\mathbf{b})$, the dry forest curve with a positive trend (spanning from 1 to $58 \%$ relative basal area) describes the observed increase in the relative abundance of Caesalpinia pyramidalis in older forests at Patos, Brazil. 
a Seasonality (rainfall CV)

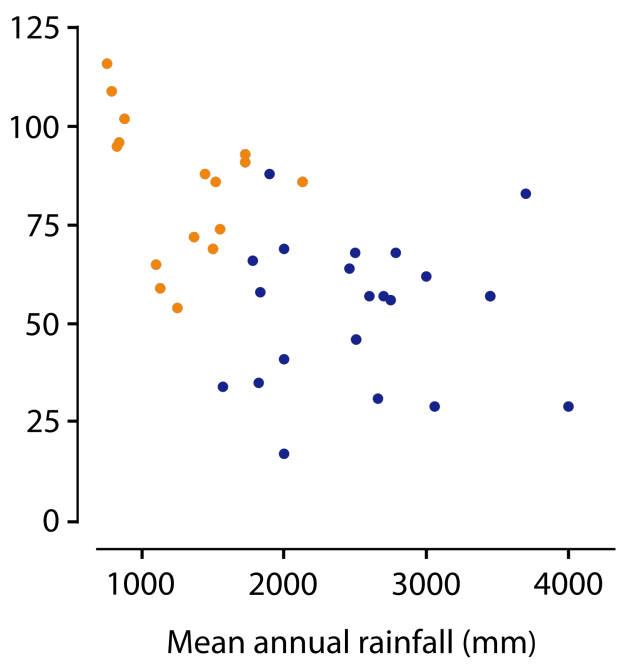

b Climatic water deficit (mm per year)

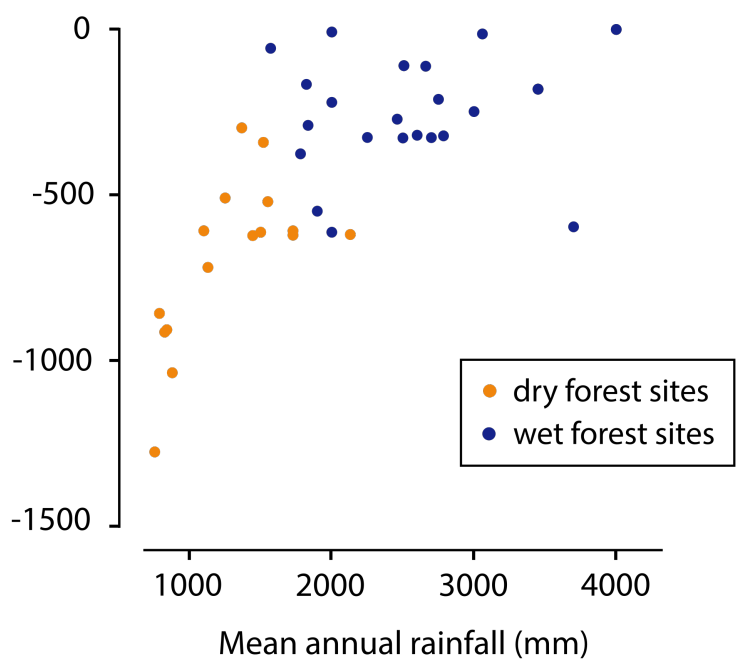

Supplementary Fig. 6 | (a) Seasonality (rainfall coefficient of variation from WorldClim $^{25}$ ), $N=41$ sites (one site was excluded because no climatic data were available), and (b) climatic water deficit (calculated as the total rainfall minus evapotranspiration during dry months and expressed in $\mathrm{mm} / \mathrm{yr}^{26}$, http://chave.upstlse.fr/pantropical_allometry.htm) in dry and wet forest sites, $N=42$ sites. 
a $\mathrm{DBH}<10 \mathrm{~cm}$

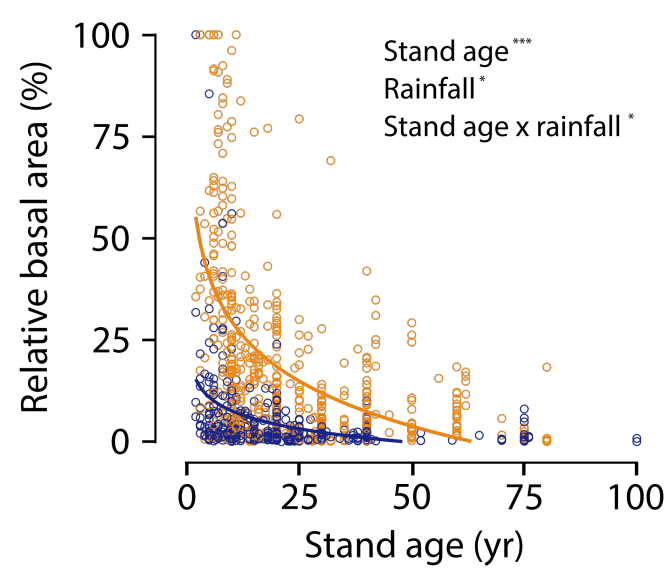

c $\mathrm{DBH} 20-30 \mathrm{~cm}$

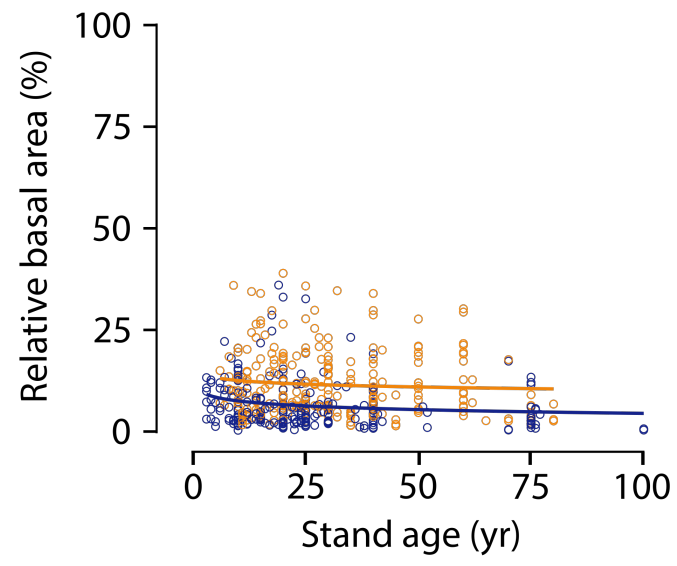

b $\mathrm{DBH} 10-20 \mathrm{~cm}$

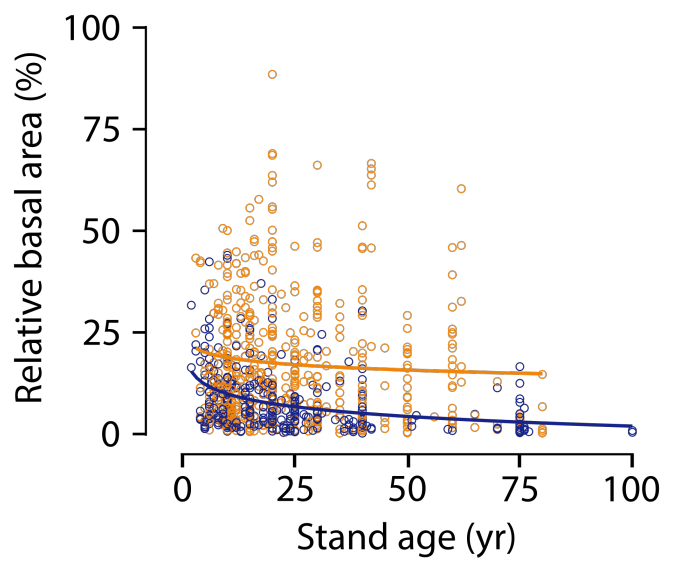

d $\mathrm{DBH}>30 \mathrm{~cm}$

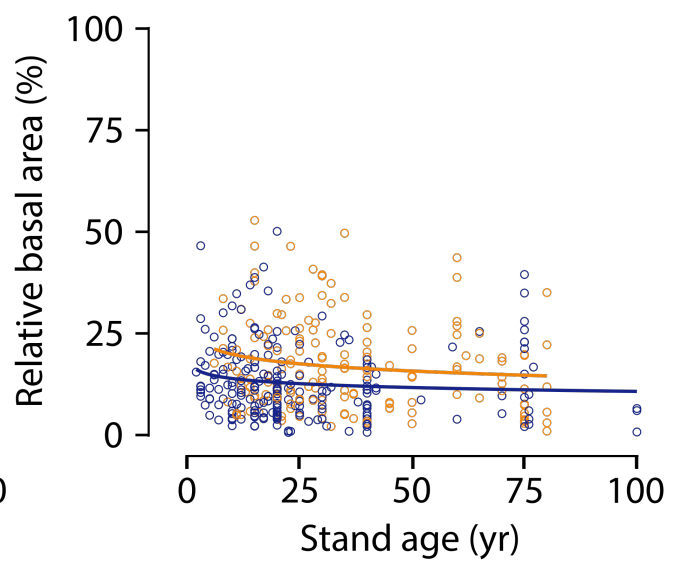

Supplementary Fig. 7 | Relationship between relative basal area of legume species and stand age in Neotropical secondary forests, $N=42$ sites.

(a) Legume species with bipinnate, (b) pinnate, and (c) unifoliolate leaves. Each line represents a different chronosequence site. 
Supplementary Table 1 | Metadata associated with 2ndFOR sites in the Neotropics.

\begin{tabular}{|c|c|c|c|c|c|c|c|c|c|c|}
\hline Site & Country & Lat. & Long. & MAT & Rainfall & Forest & CWD & Seasonality & Age & Ref. \\
\hline El Tigre & Bolivia & -11.98 & -65.72 & 26.3 & 1780 & wet & -375.63 & 66 & $3-25$ & 64 \\
\hline El Turi & Bolivia & -11.75 & -67.33 & 26 & 1833 & wet & -289.58 & 58 & $2-40$ & 64 \\
\hline San Lorenzo & Bolivia & -16.7 & -61.87 & 19.2 & 1129 & dry & -718.63 & 59 & $3-50$ & 65 \\
\hline Bahia & Brazil & -14.48 & -39.09 & 9.8 & 2000 & wet & -7.67 & 17 & $10-40$ & 66 \\
\hline Cajueiro & Brazil & -14.98 & -43.9 & 24.4 & 840 & dry & -907 & 96 & $15-59$ & \\
\hline Serra do Cipó & Brazil & -19.35 & -43.62 & 20.9 & 1519 & dry & -341 & 86 & $8-50$ & \\
\hline Eastern Pará 1 & Brazil & -4.26 & -47.73 & 25.8 & 1898 & wet & -548.79 & 88 & $5-25$ & 67 \\
\hline Eastern Pará 2 & Brazil & -2.16 & -47.38 & 26.4 & 2460 & wet & -270.92 & 64 & $5-40$ & 67 \\
\hline Eastern Pará 3 & Brazil & -1.17 & -47.75 & 26.4 & 2785 & wet & -321.25 & 68 & $2-70$ & 67 \\
\hline $\begin{array}{l}\text { Middle } \\
\text { Madeira }\end{array}$ & Brazil & -5.77 & -61.43 & 11.3 & 2507 & wet & -109.25 & 46 & $5-30$ & 68 \\
\hline $\begin{array}{l}\text { Middle } \\
\text { Madeira }\end{array}$ & Brazil & -5.77 & -61.43 & 11.3 & 2507 & wet & -109.25 & 46 & $6.5-30$ & 68 \\
\hline Mata Seca & Brazil & -14.86 & -43.99 & 24.2 & 825 & dry & -914 & 95 & $14-58$ & 69 \\
\hline Patos & Brazil & -7.02 & -37.25 & & 750 & dry & -1276 & 116 & $22-64$ & 70 \\
\hline São Paulo & Brazil & -22.32 & -47.57 & 20.4 & 1367 & dry & -297.17 & 72 & $11-45$ & 24 \\
\hline Araracuara & Colombia & -0.6 & -72.37 & 26.8 & 3059 & wet & -13.54 & 29 & $2-30$ & 71 \\
\hline Nicoya & Costa Rica & 9.97 & -85.3 & 25 & 2130 & dry & -619.32 & 86 & $5-35$ & 72 \\
\hline Matapalo & Costa Rica & 8.4 & -83.33 & 26 & 3450 & wet & -180.25 & 57 & $5-30$ & 14 \\
\hline
\end{tabular}




\begin{tabular}{|c|c|c|c|c|c|c|c|c|c|c|}
\hline Site & Country & Lat. & Long. & MAT & Rainfall & Forest & CWD & Seasonality & Age & Ref. \\
\hline Mogos & Costa Rica & 8.4 & -83.33 & 26 & 3450 & wet & -180.25 & 57 & $5-40$ & 14 \\
\hline $\begin{array}{l}\text { Santa Rosa } \\
\text { (oak forest) }\end{array}$ & Costa Rica & 10.89 & -85.6 & 25 & 1727 & dry & -608.25 & 91 & $5-70$ & 73 \\
\hline Palo Verde & Costa Rica & 10.36 & -85.31 & 26.5 & 1444 & dry & -622.5 & 88 & $7-60$ & 73 \\
\hline Piro & Costa Rica & 8.4 & -83.33 & 26 & 3450 & wet & -180.25 & 57 & $15-40$ & 14 \\
\hline Santa Rosa & Costa Rica & 10.86 & -85.61 & 24.9 & 1727 & dry & -621.25 & 93 & $6-70$ & 73 \\
\hline $\begin{array}{l}\text { Sarapiqui } \\
\text { (Chazdon) }\end{array}$ & Costa Rica & 10.43 & -83.98 & 26 & 4000 & wet & 0 & 29 & $10-41$ & 74 \\
\hline $\begin{array}{l}\text { Sarapiqui } \\
\text { (Letcher) }\end{array}$ & Costa Rica & 10.43 & -83.98 & 26 & 4000 & wet & 0 & 29 & $10-42$ & 75 \\
\hline Chajul & Mexico & 16.07 & -90.75 & 25.5 & 3000 & wet & -248 & 62 & $0-27$ & 76 \\
\hline Chamela & Mexico & 19.5 & -105.05 & 26.4 & 788 & dry & -857.54 & 109 & $3-15$ & 77 \\
\hline Chinantla & Mexico & 17.75 & -96.65 & 18.4 & 3700 & wet & -596.08 & 83 & $5-50$ & 24 \\
\hline El Ocote 1 & Mexico & 16.96 & -93.63 & 21.8 & 1500 & dry & -612.42 & 69 & $2-75$ & 78 \\
\hline El Ocote 2 & Mexico & 16.96 & -93.63 & 21.8 & 2000 & wet & -612.42 & 69 & $3-75$ & 78 \\
\hline JM Morelos & Mexico & 19.31 & -88.57 & 25.8 & 1250 & dry & -508.96 & 54 & $2-100$ & 79 \\
\hline Kaxil Kiuic & Mexico & 20.08 & -89.55 & 25.6 & 1100 & dry & -608.13 & 65 & $3-70$ & 80 \\
\hline Lacandona & Mexico & 16.54 & -90.96 & 24.6 & 2500 & wet & -327.71 & 68 & $1-31$ & 24 \\
\hline $\begin{array}{l}\text { Marqués de } \\
\text { Comillas }\end{array}$ & Mexico & 16.33 & -90.67 & & 2250 & wet & -326.21 & NA & $2-50$ & 24 \\
\hline Nizanda & Mexico & 16.66 & -95.01 & 26.2 & 878 & dry & $\begin{array}{c}- \\
1036.88\end{array}$ & 102 & $2-60$ & 81 \\
\hline Zona Norte & Mexico & 17.27 & -91.66 & 25.8 & 2750 & wet & -211 & 56 & $2-52$ & 24 \\
\hline
\end{tabular}




\begin{tabular}{lcccccccccc}
\hline Site & Country & Lat. & Long. & MAT & Rainfall & Forest & CWD & Seasonality & Age & Ref. \\
\hline Agua Salud & Panama & 9.22 & -79.78 & 26 & 2700 & wet & -326.63 & 57 & $2-31$ & 82 \\
$\begin{array}{l}\text { Barro } \\
\text { Colorado }\end{array}$ & Panama & 9.15 & -79.85 & 26 & 2600 & wet & -319.46 & 57 & $20-100$ & 83 \\
Playa Venado & Panama & 7.43 & -80.18 & 26.4 & 1550 & dry & -520.05 & 74 & $6-80$ \\
& & & & & & & & & & \\
Pucallpa & Peru & -8.5 & -74.8 & 26.2 & 1570 & wet & -56.83 & 34 & $5-30$ & 24 \\
Cayey & $\begin{array}{c}\text { Puerto } \\
\text { Rico }\end{array}$ & 18.02 & -66.08 & 21.5 & 2000 & wet & -220.17 & 41 & $10-80$ & 84 \\
El Carite & $\begin{array}{c}\text { Puerto } \\
\text { Rico }\end{array}$ & 18.08 & -66.07 & 21.5 & 1822 & wet & -165.79 & 35 & $4-77$ & 85 \\
& $\begin{array}{l}\text { Puerto } \\
\text { Luquillo }\end{array}$ & 18.34 & -65.76 & 24.4 & 2660 & wet & -111 & 31 & $9-76$ & 85 \\
\hline
\end{tabular}


Supplementary Table 2 | Taxonomic distribution ${ }^{45}$, nitrogen fixation potential, and leaf arrangement in the 398 legume species present at our 42 study sites.

\begin{tabular}{lcc}
\hline Functional trait or subfamily & $\begin{array}{c}\text { Number } \\
\text { of species }\end{array}$ & $\begin{array}{c}\text { Percentage of total species within } \\
\text { each trait or category }\end{array}$ \\
\hline
\end{tabular}

Leguminosae subfamily

Caesalpinioideae

Cercidoideae

Detarioideae

Dialioideae

Papilionoideae

Nfixation potential

Fixer

Non-fixer

Unknown fixation

Leaf type

Bipinnate

104

Pinnate

Unifoliolate

$N$-fixing species and leaf type

Fixer bipinnate

Fixer unifoliolate

Non-fixing species and leaf type

Non-fixer bipinnate

Non-fixer pinnate

Non-fixer unifoliolate

Bipinnate leaves and Nfixation potential

Bipinnate fixer

Bipinnate non-fixer

Bipinnate unknown fixation

Pinnate leaves and Nfixation potential

Pinnate fixer

Pinnate non-fixer
$54 \%$

$3 \%$

$2 \%$

$1 \%$

$40 \%$

$75 \%$

$24 \%$

$1 \%$

$26 \%$

$69 \%$

$5 \%$

$25 \%$

$73 \%$

$1 \%$

$26 \%$

$58 \%$

$16 \%$

$72 \%$

$24 \%$

$4 \%$

$80 \%$

$20 \%$

Unifoliolate leaves and Nfixation potential

Unifoliolate fixer

$21 \%$

Unifoliolate non-fixer 
Supplementary Table 3 | List of 398 Leguminosae species present in 42 Neotropical chronosequences, their current ${ }^{45}$ (and previous ${ }^{46}$ ) subfamily classification, their potential to form symbioses with N-fixing bacteria, leaf type, and average (and standard deviation) leaflet length and width $(\mathrm{cm})$.

\begin{tabular}{|c|c|c|c|c|c|}
\hline Species & Subfamily & $\begin{array}{l}\text { Potential } \\
\text { to fix } \mathrm{N}_{2}\end{array}$ & Leaf type & $\begin{array}{l}\text { Leaflet } \\
\text { length }\end{array}$ & $\begin{array}{c}\text { Leaflet } \\
\text { width }\end{array}$ \\
\hline Abarema adenophora & $\begin{array}{c}\text { Caesalpinioideae } \\
\text { (Mimosoideae) }\end{array}$ & Yes & bipinnate & $5.03 \pm 1.45$ & $3.44 \pm 1.12$ \\
\hline Abarema aggregatum & $\begin{array}{c}\text { Caesalpinioideae } \\
\text { (Mimosoideae) }\end{array}$ & Yes & bipinnate & & \\
\hline Abarema barbouriana & $\begin{array}{c}\text { Caesalpinioideae } \\
\text { (Mimosoideae) }\end{array}$ & Yes & bipinnate & $0.93 \pm 0.45$ & $0.43 \pm 0.19$ \\
\hline Abarema cochleata & $\begin{array}{c}\text { Caesalpinioideae } \\
\text { (Mimosoideae) }\end{array}$ & Yes & bipinnate & $4.84 \pm 1.26$ & $2.44 \pm 0.42$ \\
\hline Abarema jupunba & $\begin{array}{c}\text { Caesalpinioideae } \\
\text { (Mimosoideae) }\end{array}$ & Yes & bipinnate & $2.54 \pm 1.18$ & $1.5 \pm 0.7$ \\
\hline Abarema macradenia & $\begin{array}{c}\text { Caesalpinioideae } \\
\text { (Mimosoideae) }\end{array}$ & Yes & bipinnate & $2.66 \pm 0.77$ & $1.14 \pm 0.43$ \\
\hline Abarema turbinata & $\begin{array}{c}\text { Caesalpinioideae } \\
\text { (Mimosoideae) }\end{array}$ & Yes & bipinnate & $9.8 \pm 2.94$ & $3.84 \pm 1.2$ \\
\hline Acacia pennatula & $\begin{array}{c}\text { Caesalpinioideae } \\
\text { (Mimosoideae) }\end{array}$ & Yes & bipinnate & $0.18 \pm 0.04$ & $0.06 \pm 0.03$ \\
\hline Acosmium cardenasii & Papilionoideae & Yes & pinnate & $1.26 \pm 0.41$ & $0.41 \pm 0.11$ \\
\hline $\begin{array}{l}\text { Acosmium } \\
\text { lentiscifolium }\end{array}$ & Papilionoideae & Yes & pinnate & $3.23 \pm 1.75$ & $1.16 \pm 0.53$ \\
\hline Albizia adinocephala & $\begin{array}{c}\text { Caesalpinioideae } \\
\text { (Mimosoideae) }\end{array}$ & Yes & bipinnate & $4.41 \pm 1.52$ & $1.44 \pm 0.36$ \\
\hline Albizia leucocalyx & $\begin{array}{c}\text { Caesalpinioideae } \\
\text { (Mimosoideae) }\end{array}$ & Yes & bipinnate & & \\
\hline Albizia niopoides & $\begin{array}{c}\text { Caesalpinioideae } \\
\text { (Mimosoideae) }\end{array}$ & Yes & bipinnate & $0.67 \pm 0.31$ & $0.13 \pm 0.05$ \\
\hline Albizia occidentalis & $\begin{array}{c}\text { Caesalpinioideae } \\
\text { (Mimosoideae) }\end{array}$ & Yes & bipinnate & $1.8 \pm 0.39$ & $3.23 \pm 0.79$ \\
\hline Albizia polycephala & $\begin{array}{c}\text { Caesalpinioideae } \\
\text { (Mimosoideae) }\end{array}$ & Yes & bipinnate & $0.63 \pm 0.19$ & $0.27 \pm 0.09$ \\
\hline Albizia tomentosa & $\begin{array}{c}\text { Caesalpinioideae } \\
\text { (Mimosoideae) }\end{array}$ & Yes & bipinnate & $1.14 \pm 0.14$ & $0.44 \pm 0.05$ \\
\hline
\end{tabular}




\begin{tabular}{|c|c|c|c|c|c|}
\hline Species & Subfamily & $\begin{array}{l}\text { Potential } \\
\text { to fix } \mathrm{N}_{2}\end{array}$ & Leaf type & $\begin{array}{l}\text { Leaflet } \\
\text { length }\end{array}$ & $\begin{array}{c}\text { Leaflet } \\
\text { width }\end{array}$ \\
\hline Alexa grandiflora & Papilionoideae & No & pinnate & $4.95 \pm 2.28$ & $2.99 \pm 1.45$ \\
\hline Amburana cearensis & Papilionoideae & No & pinnate & $9.94 \pm 5.47$ & $4.02 \pm 1.72$ \\
\hline $\begin{array}{l}\text { Anadenanthera } \\
\text { colubrina }\end{array}$ & $\begin{array}{c}\text { Caesalpinioideae } \\
\text { (Mimosoideae) }\end{array}$ & Yes & bipinnate & $0.48 \pm 0.14$ & $0.13 \pm 0.04$ \\
\hline Andira inermis & Papilionoideae & Yes & pinnate & $7.23 \pm 1.14$ & $2.66 \pm 0.63$ \\
\hline Andira legalis & Papilionoideae & Yes & pinnate & $10.95 \pm 3.27$ & $5.04 \pm 1.93$ \\
\hline Andira lewisii & Papilionoideae & Yes & pinnate & & \\
\hline Andira multistipula & Papilionoideae & Yes & pinnate & $7.97 \pm 3.15$ & $2.73 \pm 1.04$ \\
\hline Andira parviflora & Papilionoideae & Yes & pinnate & $3.9 \pm 1.14$ & $1.76 \pm 0.34$ \\
\hline Apoplanesia paniculata & Papilionoideae & No & pinnate & $3.35 \pm 1.11$ & $1.29 \pm 0.25$ \\
\hline Apuleia leiocarpa & $\begin{array}{c}\text { Dialioideae } \\
\text { (Caesalpinioideae) }\end{array}$ & No & pinnate & $3.39 \pm 0.86$ & $1.71 \pm 0.33$ \\
\hline Arapatiella psilophylla & Caesalpinioideae & No & pinnate & $7.89 \pm 1.44$ & $3.51 \pm 0.66$ \\
\hline Ateleia cubensis & Papilionoideae & Yes & pinnate & $3.22 \pm 0.97$ & $1.52 \pm 0.27$ \\
\hline Ateleia herbert-smithii & Papilionoideae & Yes & pinnate & $6.98 \pm 1.09$ & $3.46 \pm 0.45$ \\
\hline Balizia elegans & $\begin{array}{c}\text { Caesalpinioideae } \\
\text { (Mimosoideae) }\end{array}$ & Yes & bipinnate & $0.45 \pm 0.08$ & $0.15 \pm 0.03$ \\
\hline Balizia leucocalix & $\begin{array}{c}\text { Caesalpinioideae } \\
\text { (Mimosoideae) }\end{array}$ & Yes & bipinnate & $2.57 \pm 0.54$ & $1.37 \pm 0.37$ \\
\hline Balizia pedicellaris & $\begin{array}{c}\text { Caesalpinioideae } \\
\text { (Mimosoideae) }\end{array}$ & Yes & bipinnate & $0.67 \pm 0.13$ & $0.21 \pm 0.04$ \\
\hline Batesia floribunda & Caesalpinioideae & No & pinnate & $11.35 \pm 2.51$ & $4.87 \pm 0.92$ \\
\hline Bauhinia acreana & $\begin{array}{c}\text { Cercidoideae } \\
\text { (Caesalpinioideae) }\end{array}$ & No & unifoliolate & $7.7 \pm 1.13$ & $6.02 \pm 1.56$ \\
\hline Bauhinia acuruana & $\begin{array}{c}\text { Cercidoideae } \\
\text { (Caesalpinioideae) }\end{array}$ & No & unifoliolate & $6.21 \pm 2.4$ & $4.03 \pm 1.12$ \\
\hline Bauhinia brevipes & $\begin{array}{c}\text { Cercidoideae } \\
\text { (Caesalpinioideae) }\end{array}$ & No & unifoliolate & $4.31 \pm 0.95$ & $2.32 \pm 0.49$ \\
\hline Bauhinia cheilantha & $\begin{array}{c}\text { Cercidoideae } \\
\text { (Caesalpinioideae) }\end{array}$ & No & unifoliolate & $7.72 \pm 1.78$ & $7.6 \pm 1.4$ \\
\hline Bauhinia divaricata & $\begin{array}{c}\text { Cercidoideae } \\
\text { (Caesalpinioideae) }\end{array}$ & No & unifoliolate & $5.87 \pm 2.97$ & $6.51 \pm 1.54$ \\
\hline Bauhinia guianensis & $\begin{array}{c}\text { Cercidoideae } \\
\text { (Caesalpinioideae) }\end{array}$ & No & bifoliolate & $11.19 \pm 3.93$ & $3.79 \pm 0.59$ \\
\hline
\end{tabular}




\begin{tabular}{|c|c|c|c|c|c|}
\hline Species & Subfamily & $\begin{array}{l}\text { Potential } \\
\text { to fix } \mathrm{N}_{2}\end{array}$ & Leaf type & $\begin{array}{l}\text { Leaflet } \\
\text { length }\end{array}$ & $\begin{array}{l}\text { Leaflet } \\
\text { width }\end{array}$ \\
\hline Bauhinia longicuspis & $\begin{array}{c}\text { Cercidoideae } \\
\text { (Caesalpinioideae) }\end{array}$ & No & unifoliolate & $12.93 \pm 1.74$ & $6 \pm 2.23$ \\
\hline $\begin{array}{l}\text { Bauhinia } \\
\text { melastomatoides }\end{array}$ & $\begin{array}{c}\text { Cercidoideae } \\
\text { (Caesalpinioideae) }\end{array}$ & No & unifoliolate & $17.47 \pm 1.71$ & $7.33 \pm 1.04$ \\
\hline Bauhinia ovata & $\begin{array}{c}\text { Cercidoideae } \\
\text { (Caesalpinioideae) }\end{array}$ & No & unifoliolate & $7.72 \pm 0.9$ & $4.83 \pm 0.9$ \\
\hline Bauhinia pauletia & $\begin{array}{c}\text { Cercidoideae } \\
\text { (Caesalpinioideae) }\end{array}$ & No & unifoliolate & $4.04 \pm 1.08$ & $4.23 \pm 1.46$ \\
\hline Bauhinia rufa & $\begin{array}{c}\text { Cercidoideae } \\
\text { (Caesalpinioideae) }\end{array}$ & No & unifoliolate & $8.42 \pm 2.34$ & $7.64 \pm 1.81$ \\
\hline Bauhinia ungulata & $\begin{array}{c}\text { Cercidoideae } \\
\text { (Caesalpinioideae) }\end{array}$ & No & unifoliolate & $8.42 \pm 4.32$ & $6.17 \pm 3.06$ \\
\hline Bowdichia virgilioides & Papilionoideae & Yes & pinnate & $3.36 \pm 0.82$ & $1.42 \pm 0.56$ \\
\hline Caesalpinia caladenia & Caesalpinioideae & No & bipinnate & $1.67 \pm 0.45$ & $0.92 \pm 0.26$ \\
\hline Caesalpinia coriaria & Caesalpinioideae & No & bipinnate & $0.45 \pm 0.08$ & $0.15 \pm 0.02$ \\
\hline Caesalpinia eriostachys & Caesalpinioideae & No & bipinnate & $0.95 \pm 0.21$ & $0.45 \pm 0.06$ \\
\hline Caesalpinia exostemma & Caesalpinioideae & No & bipinnate & $1.73 \pm 0.44$ & $0.86 \pm 0.17$ \\
\hline Caesalpinia ferrea & Caesalpinioideae & No & bipinnate & $1.66 \pm 0.51$ & $0.89 \pm 0.26$ \\
\hline Caesalpinia floribunda & Caesalpinioideae & No & bipinnate & $0.72 \pm 0.13$ & $0.4 \pm 0.04$ \\
\hline Caesalpinia gaumeri & Caesalpinioideae & No & bipinnate & $1.68 \pm 0.35$ & $0.98 \pm 0.22$ \\
\hline Caesalpinia mollis & Caesalpinioideae & No & bipinnate & $4.46 \pm 1.32$ & $1.89 \pm 0.27$ \\
\hline Caesalpinia platyloba & Caesalpinioideae & No & bipinnate & $3.35 \pm 1.06$ & $1.55 \pm 0.2$ \\
\hline $\begin{array}{l}\text { Caesalpinia } \\
\text { pyramidalis }\end{array}$ & Caesalpinioideae & No & bipinnate & $1.96 \pm 0.36$ & $1.22 \pm 0.17$ \\
\hline $\begin{array}{l}\text { Caesalpinia } \\
\text { sclerocarpa }\end{array}$ & Caesalpinioideae & No & bipinnate & $1.61 \pm 0.56$ & $0.87 \pm 0.32$ \\
\hline $\begin{array}{l}\text { Caesalpinia } \\
\text { yucatanensis }\end{array}$ & Caesalpinioideae & No & bipinnate & $3.08 \pm 0.86$ & $1.46 \pm 0.44$ \\
\hline Calliandra formosa & $\begin{array}{c}\text { Caesalpinioideae } \\
\text { (Mimosoideae) }\end{array}$ & Yes & bipinnate & $0.69 \pm 0.23$ & $0.31 \pm 0.08$ \\
\hline Calliandra grandiflora & $\begin{array}{c}\text { Caesalpinioideae } \\
\text { (Mimosoideae) }\end{array}$ & Yes & bipinnate & $0.39 \pm 0.18$ & $0.11 \pm 0.04$ \\
\hline Cassia fastuosa & Caesalpinioideae & No & pinnate & $3.78 \pm 0.8$ & $1.01 \pm 0.15$ \\
\hline Cassia ferruginea & Caesalpinioideae & No & pinnate & $2.74 \pm 0.5$ & $0.86 \pm 0.09$ \\
\hline Cassia grandis & Caesalpinioideae & No & pinnate & $4.04 \pm 1.47$ & $1.39 \pm 0.23$ \\
\hline
\end{tabular}




\begin{tabular}{|c|c|c|c|c|c|}
\hline Species & Subfamily & $\begin{array}{l}\text { Potential } \\
\text { to fix } \mathrm{N}_{2}\end{array}$ & Leaf type & $\begin{array}{l}\text { Leaflet } \\
\text { length }\end{array}$ & $\begin{array}{l}\text { Leaflet } \\
\text { width }\end{array}$ \\
\hline Cenostigma tocantinum & Caesalpinioideae & No & pinnate & $9.55 \pm 2.83$ & $3.67 \pm 0.76$ \\
\hline $\begin{array}{l}\text { Centrolobium } \\
\text { microchaete }\end{array}$ & Papilionoideae & Yes & pinnate & $5.53 \pm 1.88$ & $4.08 \pm 2.36$ \\
\hline $\begin{array}{l}\text { Centrolobium } \\
\text { sclerophyllum }\end{array}$ & Papilionoideae & Yes & pinnate & $7.48 \pm 1.91$ & $4.38 \pm 0.9$ \\
\hline $\begin{array}{l}\text { Centrolobium } \\
\text { tomentosum }\end{array}$ & Papilionoideae & Yes & pinnate & $9.75 \pm 2.28$ & $5.21 \pm 1.33$ \\
\hline Ceratonia siliqua & Caesalpinioideae & No & pinnate & $4.82 \pm 0.81$ & $3.17 \pm 0.54$ \\
\hline $\begin{array}{l}\text { Chamaecrista } \\
\text { apoucouita }\end{array}$ & Caesalpinioideae & Yes & pinnate & $5.76 \pm 1.27$ & $2.56 \pm 0.6$ \\
\hline Chamaecrista bahiae & Caesalpinioideae & Yes & pinnate & $7.63 \pm 1.89$ & $4.36 \pm 0.8$ \\
\hline Chamaecrista duartei & Caesalpinioideae & Yes & pinnate & $9.94 \pm 2.64$ & $4.37 \pm 0.8$ \\
\hline $\begin{array}{l}\text { Chamaecrista } \\
\text { eitenorum }\end{array}$ & Caesalpinioideae & Yes & pinnate & $5.47 \pm 1.28$ & $2.77 \pm 0.67$ \\
\hline $\begin{array}{l}\text { Chamaecrista } \\
\text { xinguensis }\end{array}$ & Caesalpinioideae & Yes & pinnate & $5.91 \pm 2.23$ & $2.46 \pm 1$ \\
\hline Chloroleucon dumosum & $\begin{array}{c}\text { Caesalpinioideae } \\
\text { (Mimosoideae) }\end{array}$ & Yes & bipinnate & $0.77 \pm 0.19$ & $0.26 \pm 0.1$ \\
\hline $\begin{array}{l}\text { Chloroleucon } \\
\text { foliolosum }\end{array}$ & $\begin{array}{c}\text { Caesalpinioideae } \\
\text { (Mimosoideae) }\end{array}$ & Yes & bipinnate & $0.22 \pm 0.1$ & $0.07 \pm 0.02$ \\
\hline $\begin{array}{l}\text { Chloroleucon } \\
\text { mangense }\end{array}$ & $\begin{array}{c}\text { Caesalpinioideae } \\
\text { (Mimosoideae) }\end{array}$ & Yes & bipinnate & $0.72 \pm 0.26$ & $0.29 \pm 0.18$ \\
\hline $\begin{array}{l}\text { Clathrotropis } \\
\text { macrocarpa }\end{array}$ & Papilionoideae & Yes & pinnate & $12.6 \pm 4.63$ & $5.14 \pm 1.34$ \\
\hline Clitoria glaberrima & Papilionoideae & Yes & pinnate & $10.06 \pm 3.22$ & $5.26 \pm 1.55$ \\
\hline Cojoba arborea & $\begin{array}{l}\text { Caesalpinioideae } \\
\text { (Mimosoideae) }\end{array}$ & Yes & bipinnate & $0.59 \pm 0.18$ & $0.13 \pm 0.03$ \\
\hline Copaifera langsdorffii & $\begin{array}{c}\text { Detarioideae } \\
\text { (Caesalpinioideae) }\end{array}$ & No & pinnate & $3.18 \pm 1$ & $1.65 \pm 0.44$ \\
\hline Coursertia ferruginea & Papilionoideae & Yes & pinnate & $3.25 \pm 0.94$ & $1.87 \pm 0.28$ \\
\hline Crotalaria maypurensis & Papilionoideae & Yes & pinnate & $4.85 \pm 1.33$ & $1.54 \pm 0.32$ \\
\hline Crudia amazonica & $\begin{array}{c}\text { Detarioideae } \\
\text { (Caesalpinioideae) }\end{array}$ & No & pinnate & $5.37 \pm 1.47$ & $1.64 \pm 0.34$ \\
\hline Dalbergia acuta & Papilionoideae & Yes & pinnate & $1.81 \pm 0.6$ & $0.63 \pm 0.15$ \\
\hline Dalbergia brownei & Papilionoideae & Yes & pinnate & $3.99 \pm 1.2$ & $2.78 \pm 1.18$ \\
\hline
\end{tabular}




\begin{tabular}{|c|c|c|c|c|c|}
\hline Species & Subfamily & $\begin{array}{l}\text { Potential } \\
\text { to fix } \mathrm{N}_{2}\end{array}$ & Leaf type & $\begin{array}{l}\text { Leaflet } \\
\text { length }\end{array}$ & $\begin{array}{l}\text { Leaflet } \\
\text { width }\end{array}$ \\
\hline Dalbergia cearensis & Papilionoideae & Yes & pinnate & $3.41 \pm 0.99$ & $1.77 \pm 0.63$ \\
\hline Dalbergia congestiflora & Papilionoideae & Yes & pinnate & $2.97 \pm 0.95$ & $1.6 \pm 0.54$ \\
\hline Dalbergia glomerata & Papilionoideae & Yes & pinnate & $4.32 \pm 1.19$ & $1.92 \pm 0.44$ \\
\hline Dalbergia monetaria & Papilionoideae & Yes & pinnate & $8.05 \pm 3.1$ & $4.86 \pm 1.19$ \\
\hline Dalbergia retusa & Papilionoideae & Yes & pinnate & $6.36 \pm 2.1$ & $3.11 \pm 1.18$ \\
\hline Dalbergia spruceana & Papilionoideae & Yes & pinnate & $2.96 \pm 0.88$ & $1.26 \pm 0.22$ \\
\hline Dalbergia stevensonii & Papilionoideae & Yes & pinnate & $6.46 \pm 0.47$ & $3.08 \pm 0.21$ \\
\hline Dialium guianense & $\begin{array}{c}\text { Dialioideae } \\
\text { (Caesalpinioideae) }\end{array}$ & No & pinnate & $7.94 \pm 3.08$ & $3.45 \pm 0.74$ \\
\hline Diphysa americana & Papilionoideae & Yes & pinnate & $2.49 \pm 0.48$ & $1.33 \pm 0.19$ \\
\hline Diphysa carthagenensis & Papilionoideae & Yes & pinnate & $1.04 \pm 0.3$ & $0.51 \pm 0.2$ \\
\hline Diphysa robinioides & Papilionoideae & Yes & pinnate & $2.19 \pm 0.43$ & $0.8 \pm 0.11$ \\
\hline Diphysa yucatanensis & Papilionoideae & Yes & pinnate & $3.16 \pm 0.84$ & $1.74 \pm 0.5$ \\
\hline Diplotropis ferruginea & Papilionoideae & Yes & pinnate & $5.35 \pm 1.63$ & $2.68 \pm 0.34$ \\
\hline Diplotropis incexis & Papilionoideae & Yes & pinnate & $5.87 \pm 1.3$ & $2.79 \pm 0.25$ \\
\hline Diplotropis martiusii & Papilionoideae & Yes & pinnate & $10.51 \pm 3.01$ & $4.34 \pm 1.15$ \\
\hline Diplotropis purpurea & Papilionoideae & Yes & pinnate & $7.89 \pm 2.31$ & $4.49 \pm 0.97$ \\
\hline Diplotropis triloba & Papilionoideae & Yes & pinnate & $8.02 \pm 1.37$ & $4.17 \pm 0.82$ \\
\hline Dipteryx odorata & Papilionoideae & No & pinnate & $13.37 \pm 2.89$ & $5.68 \pm 0.78$ \\
\hline Dipteryx oleifera & Papilionoideae & No & pinnate & & \\
\hline Dipteryx panamensis & Papilionoideae & No & pinnate & $11.47 \pm 1.73$ & $5.14 \pm 0.58$ \\
\hline $\begin{array}{l}\text { Diptychandra } \\
\text { aurantiaca }\end{array}$ & Caesalpinioideae & No & pinnate & $4.16 \pm 1.06$ & $1.89 \pm 0.39$ \\
\hline $\begin{array}{l}\text { Dussia } \\
\text { macroprophyllata }\end{array}$ & Papilionoideae & Yes & pinnate & $9.98 \pm 2.63$ & $5.93 \pm 1.09$ \\
\hline $\begin{array}{l}\text { Enterolobium } \\
\text { contortisiliquum }\end{array}$ & $\begin{array}{l}\text { Caesalpinioideae } \\
\text { (Mimosoideae) }\end{array}$ & Yes & bipinnate & $1.62 \pm 0.66$ & $0.53 \pm 0.21$ \\
\hline $\begin{array}{l}\text { Enterolobium } \\
\text { cyclocarpum }\end{array}$ & $\begin{array}{l}\text { Caesalpinioideae } \\
\text { (Mimosoideae) }\end{array}$ & Yes & bipinnate & $0.93 \pm 0.28$ & $0.25 \pm 0.05$ \\
\hline $\begin{array}{l}\text { Enterolobium } \\
\text { schomburgkii }\end{array}$ & $\begin{array}{l}\text { Caesalpinioideae } \\
\text { (Mimosoideae) }\end{array}$ & Yes & bipinnate & $0.25 \pm 0.08$ & $0.05 \pm 0.02$ \\
\hline Erythrina berteroana & Papilionoideae & Yes & pinnate & $8.06 \pm 0.75$ & $5.22 \pm 1.13$ \\
\hline Erythrina costaricensis & Papilionoideae & Yes & pinnate & $9.02 \pm 0.31$ & $5.66 \pm 0.84$ \\
\hline Erythrina folkersii & Papilionoideae & Yes & pinnate & $7.76 \pm 2.32$ & $6.84 \pm 1.39$ \\
\hline Erythrina standleyana & Papilionoideae & Yes & pinnate & & \\
\hline
\end{tabular}




\begin{tabular}{|c|c|c|c|c|c|}
\hline Species & Subfamily & $\begin{array}{l}\text { Potential } \\
\text { to fix } \mathrm{N}_{2}\end{array}$ & Leaf type & $\begin{array}{l}\text { Leaflet } \\
\text { length }\end{array}$ & $\begin{array}{l}\text { Leaflet } \\
\text { width }\end{array}$ \\
\hline Exostyles venusta & Papilionoideae & No & pinnate & $2.95 \pm 1.09$ & $1.38 \pm 0.29$ \\
\hline Gliricidia maculata & Papilionoideae & Yes & pinnate & & \\
\hline Gliricidia sepium & Papilionoideae & Yes & pinnate & $5.4 \pm 1.38$ & $2.79 \pm 0.84$ \\
\hline $\begin{array}{l}\text { Goniorrhachis } \\
\text { marginata }\end{array}$ & $\begin{array}{c}\text { Detarioideae } \\
\text { (Caesalpinioideae) }\end{array}$ & No & pinnate & $4.13 \pm 1.05$ & $1.85 \pm 0.23$ \\
\hline $\begin{array}{l}\text { Haematoxylum } \\
\text { brasiletto }\end{array}$ & Caesalpinioideae & No & pinnate & $2.59 \pm 3.61$ & $0.72 \pm 0.12$ \\
\hline $\begin{array}{l}\text { Haematoxylum } \\
\text { campechianum }\end{array}$ & Caesalpinioideae & No & pinnate & $1.67 \pm 0.28$ & $1.4 \pm 0.16$ \\
\hline $\begin{array}{l}\text { Harleyodendron } \\
\text { unifoliolatum }\end{array}$ & Papilionoideae & No & unifoliolate & $26.31 \pm 5.4$ & $7.51 \pm 1.27$ \\
\hline Havardia albicans & $\begin{array}{l}\text { Caesalpinioideae } \\
\text { (Mimosoideae) }\end{array}$ & Yes & bipinnate & $0.59 \pm 0.12$ & $0.15 \pm 0.02$ \\
\hline $\begin{array}{l}\text { Havardia } \\
\text { campylacantha }\end{array}$ & $\begin{array}{c}\text { Caesalpinioideae } \\
\text { (Mimosoideae) }\end{array}$ & Yes & bipinnate & $0.26 \pm 0.08$ & $0.07 \pm 0.03$ \\
\hline Hymenaea courbaril & $\begin{array}{c}\text { Detarioideae } \\
\text { (Caesalpinioideae) }\end{array}$ & No & pinnate & $7.32 \pm 1.19$ & $3.14 \pm 0.3$ \\
\hline Hymenaea parvifolia & $\begin{array}{c}\text { Detarioideae } \\
\text { (Caesalpinioideae) }\end{array}$ & No & pinnate & $6.97 \pm 1.4$ & $3.46 \pm 0.8$ \\
\hline $\begin{array}{l}\text { Hymenolobium } \\
\text { mesoamericanum }\end{array}$ & Papilionoideae & Yes & pinnate & $4.56 \pm 1.42$ & $1.99 \pm 0.45$ \\
\hline Indigofera fruticosa & Papilionoideae & Yes & pinnate & $2.12 \pm 0.24$ & $1.16 \pm 0.22$ \\
\hline Inga acrocephala & $\begin{array}{l}\text { Caesalpinioideae } \\
\text { (Mimosoideae) }\end{array}$ & Yes & pinnate & $10.52 \pm 4$ & $3.99 \pm 1.16$ \\
\hline Inga acuminata & $\begin{array}{l}\text { Caesalpinioideae } \\
\text { (Mimosoideae) }\end{array}$ & Yes & pinnate & $9.07 \pm 2.82$ & $2.76 \pm 0.88$ \\
\hline Inga aestuariorum & $\begin{array}{l}\text { Caesalpinioideae } \\
\text { (Mimosoideae) }\end{array}$ & Yes & pinnate & & \\
\hline Inga alba & $\begin{array}{l}\text { Caesalpinioideae } \\
\text { (Mimosoideae) }\end{array}$ & Yes & pinnate & $5.92 \pm 2.86$ & $4.12 \pm 3.32$ \\
\hline Inga auristellae & $\begin{array}{l}\text { Caesalpinioideae } \\
\text { (Mimosoideae) }\end{array}$ & Yes & pinnate & $5.66 \pm 2.69$ & $2.62 \pm 0.81$ \\
\hline Inga bella & $\begin{array}{c}\text { Caesalpinioideae } \\
\text { (Mimosoideae) }\end{array}$ & Yes & pinnate & $15.53 \pm 3.74$ & $6.82 \pm 1.55$ \\
\hline
\end{tabular}




\begin{tabular}{|c|c|c|c|c|c|}
\hline Species & Subfamily & $\begin{array}{l}\text { Potential } \\
\text { to fix } \mathrm{N}_{2}\end{array}$ & Leaf type & $\begin{array}{l}\text { Leaflet } \\
\text { length }\end{array}$ & $\begin{array}{l}\text { Leaflet } \\
\text { width }\end{array}$ \\
\hline Inga blanchetiana & $\begin{array}{c}\text { Caesalpinioideae } \\
\text { (Mimosoideae) }\end{array}$ & Yes & pinnate & $10.57 \pm 3.45$ & $2.15 \pm 0.69$ \\
\hline Inga brachyrhachis & $\begin{array}{c}\text { Caesalpinioideae } \\
\text { (Mimosoideae) }\end{array}$ & Yes & pinnate & $15.03 \pm 4.4$ & $6.79 \pm 1.8$ \\
\hline Inga capitata & $\begin{array}{c}\text { Caesalpinioideae } \\
\text { (Mimosoideae) }\end{array}$ & Yes & pinnate & $8.3 \pm 3.78$ & $3.38 \pm 1.56$ \\
\hline Inga cayennensis & $\begin{array}{c}\text { Caesalpinioideae } \\
\text { (Mimosoideae) }\end{array}$ & Yes & pinnate & $8.09 \pm 3.19$ & $3.6 \pm 1.27$ \\
\hline Inga chartacea & $\begin{array}{c}\text { Caesalpinioideae } \\
\text { (Mimosoideae) }\end{array}$ & Yes & pinnate & $16.51 \pm 3.65$ & $7.45 \pm 1.97$ \\
\hline Inga chocoensis & $\begin{array}{c}\text { Caesalpinioideae } \\
\text { (Mimosoideae) }\end{array}$ & Yes & pinnate & $15.77 \pm 7.06$ & $9.35 \pm 3.45$ \\
\hline Inga chrysantha & $\begin{array}{c}\text { Caesalpinioideae } \\
\text { (Mimosoideae) }\end{array}$ & Yes & pinnate & $10.08 \pm 3.48$ & $3.19 \pm 1.04$ \\
\hline Inga cinnamomea & $\begin{array}{c}\text { Caesalpinioideae } \\
\text { (Mimosoideae) }\end{array}$ & Yes & pinnate & $12.2 \pm 3.27$ & $5.02 \pm 0.95$ \\
\hline Inga cocleensis & $\begin{array}{c}\text { Caesalpinioideae } \\
\text { (Mimosoideae) }\end{array}$ & Yes & pinnate & $11.54 \pm 4.08$ & $4.27 \pm 1.13$ \\
\hline Inga coruscans & $\begin{array}{c}\text { Caesalpinioideae } \\
\text { (Mimosoideae) }\end{array}$ & Yes & pinnate & $9.82 \pm 4.5$ & $4.29 \pm 1.77$ \\
\hline Inga disticha & $\begin{array}{c}\text { Caesalpinioideae } \\
\text { (Mimosoideae) }\end{array}$ & Yes & pinnate & $7.85 \pm 2.68$ & $2.87 \pm 0.74$ \\
\hline Inga edulis & $\begin{array}{c}\text { Caesalpinioideae } \\
\text { (Mimosoideae) }\end{array}$ & Yes & pinnate & $8.89 \pm 3.51$ & $4.1 \pm 1.85$ \\
\hline Inga filiformis & $\begin{array}{c}\text { Caesalpinioideae } \\
\text { (Mimosoideae) }\end{array}$ & Yes & pinnate & $17.57 \pm 6.36$ & $7.53 \pm 1.78$ \\
\hline Inga flagelliformis & $\begin{array}{c}\text { Caesalpinioideae } \\
\text { (Mimosoideae) }\end{array}$ & Yes & pinnate & $11.28 \pm 2.27$ & $3.46 \pm 0.77$ \\
\hline Inga goldmanii & $\begin{array}{c}\text { Caesalpinioideae } \\
\text { (Mimosoideae) }\end{array}$ & Yes & pinnate & & \\
\hline Inga gracilifolia & $\begin{array}{c}\text { Caesalpinioideae } \\
\text { (Mimosoideae) }\end{array}$ & Yes & pinnate & $2.81 \pm 0.7$ & $1.11 \pm 0.29$ \\
\hline Inga grandiflora & $\begin{array}{c}\text { Caesalpinioideae } \\
\text { (Mimosoideae) }\end{array}$ & Yes & pinnate & $13.92 \pm 4.39$ & $5.55 \pm 1.73$ \\
\hline
\end{tabular}




\begin{tabular}{|c|c|c|c|c|c|}
\hline Species & Subfamily & $\begin{array}{l}\text { Potential } \\
\text { to fix } \mathrm{N}_{2}\end{array}$ & Leaf type & $\begin{array}{l}\text { Leaflet } \\
\text { length }\end{array}$ & $\begin{array}{l}\text { Leaflet } \\
\text { width }\end{array}$ \\
\hline Inga hayesii & $\begin{array}{c}\text { Caesalpinioideae } \\
\text { (Mimosoideae) }\end{array}$ & Yes & pinnate & $7.41 \pm 1.9$ & $3.56 \pm 0.67$ \\
\hline Inga heterophylla & $\begin{array}{c}\text { Caesalpinioideae } \\
\text { (Mimosoideae) }\end{array}$ & Yes & pinnate & $4.89 \pm 1.19$ & $1.72 \pm 0.41$ \\
\hline Inga ingoides & $\begin{array}{c}\text { Caesalpinioideae } \\
\text { (Mimosoideae) }\end{array}$ & Yes & pinnate & $9.32 \pm 2.33$ & $4.59 \pm 1.2$ \\
\hline Inga jimenezii & $\begin{array}{c}\text { Caesalpinioideae } \\
\text { (Mimosoideae) }\end{array}$ & Yes & pinnate & $18.25 \pm 5.89$ & $8.09 \pm 2.03$ \\
\hline Inga jinicuil & $\begin{array}{c}\text { Caesalpinioideae } \\
\text { (Mimosoideae) }\end{array}$ & Yes & pinnate & $8.33 \pm 3.2$ & $3.4 \pm 1.22$ \\
\hline Inga lateriflora & $\begin{array}{c}\text { Caesalpinioideae } \\
\text { (Mimosoideae) }\end{array}$ & Yes & pinnate & $5.89 \pm 1.42$ & $2.33 \pm 0.69$ \\
\hline Inga latibracteata & $\begin{array}{c}\text { Caesalpinioideae } \\
\text { (Mimosoideae) }\end{array}$ & Yes & pinnate & $8.55 \pm 3.93$ & $4.33 \pm 1.78$ \\
\hline Inga laurina & $\begin{array}{c}\text { Caesalpinioideae } \\
\text { (Mimosoideae) }\end{array}$ & Yes & pinnate & $6.12 \pm 3$ & $2.76 \pm 1.28$ \\
\hline Inga leiocalycina & $\begin{array}{c}\text { Caesalpinioideae } \\
\text { (Mimosoideae) }\end{array}$ & Yes & pinnate & $8.15 \pm 3.8$ & $3.38 \pm 1.36$ \\
\hline Inga litoralis & $\begin{array}{c}\text { Caesalpinioideae } \\
\text { (Mimosoideae) }\end{array}$ & Yes & pinnate & $9.82 \pm 3.03$ & $4.17 \pm 0.93$ \\
\hline Inga lomatophylla & $\begin{array}{c}\text { Caesalpinioideae } \\
\text { (Mimosoideae) }\end{array}$ & Yes & pinnate & $13.28 \pm 4.06$ & $6.92 \pm 2.02$ \\
\hline Inga longiflora & $\begin{array}{c}\text { Caesalpinioideae } \\
\text { (Mimosoideae) }\end{array}$ & Yes & pinnate & $12.71 \pm 4.43$ & $4.07 \pm 1.01$ \\
\hline Inga luschnathiana & $\begin{array}{c}\text { Caesalpinioideae } \\
\text { (Mimosoideae) }\end{array}$ & Yes & pinnate & $5.17 \pm 2.08$ & $5.47 \pm 4.1$ \\
\hline Inga macrophylla & $\begin{array}{c}\text { Caesalpinioideae } \\
\text { (Mimosoideae) }\end{array}$ & Yes & pinnate & $12.34 \pm 3.13$ & $6.38 \pm 1.48$ \\
\hline Inga marginata & $\begin{array}{c}\text { Caesalpinioideae } \\
\text { (Mimosoideae) }\end{array}$ & Yes & pinnate & $9.12 \pm 3.25$ & $2.96 \pm 0.65$ \\
\hline Inga micheliana & $\begin{array}{c}\text { Caesalpinioideae } \\
\text { (Mimosoideae) }\end{array}$ & Yes & pinnate & $7.33 \pm 2.98$ & $3.03 \pm 1.43$ \\
\hline Inga microcalyx & $\begin{array}{c}\text { Caesalpinioideae } \\
\text { (Mimosoideae) }\end{array}$ & Yes & pinnate & $9.76 \pm 3.6$ & $5.87 \pm 1.92$ \\
\hline
\end{tabular}




\begin{tabular}{|c|c|c|c|c|c|}
\hline Species & Subfamily & $\begin{array}{l}\text { Potential } \\
\text { to fix } \mathrm{N}_{2}\end{array}$ & Leaf type & $\begin{array}{l}\text { Leaflet } \\
\text { length }\end{array}$ & $\begin{array}{l}\text { Leaflet } \\
\text { width }\end{array}$ \\
\hline Inga mucuna & $\begin{array}{c}\text { Caesalpinioideae } \\
\text { (Mimosoideae) }\end{array}$ & Yes & pinnate & $12.64 \pm 2.17$ & $6 \pm 1.14$ \\
\hline Inga multijuga & $\begin{array}{c}\text { Caesalpinioideae } \\
\text { (Mimosoideae) }\end{array}$ & Yes & pinnate & $9.12 \pm 4.88$ & $3.52 \pm 1.49$ \\
\hline Inga nobilis & $\begin{array}{c}\text { Caesalpinioideae } \\
\text { (Mimosoideae) }\end{array}$ & Yes & pinnate & $7.68 \pm 2.34$ & $2.94 \pm 1.13$ \\
\hline Inga obidensis & $\begin{array}{c}\text { Caesalpinioideae } \\
\text { (Mimosoideae) }\end{array}$ & Yes & pinnate & $9.36 \pm 2.54$ & $4.16 \pm 0.84$ \\
\hline Inga oerstediana & $\begin{array}{c}\text { Caesalpinioideae } \\
\text { (Mimosoideae) }\end{array}$ & Yes & pinnate & $9.23 \pm 3.17$ & $4.63 \pm 2.39$ \\
\hline Inga paraensis & $\begin{array}{c}\text { Caesalpinioideae } \\
\text { (Mimosoideae) }\end{array}$ & Yes & pinnate & $10.18 \pm 3.2$ & $4.99 \pm 1.18$ \\
\hline Inga pauciflora & $\begin{array}{c}\text { Caesalpinioideae } \\
\text { (Mimosoideae) }\end{array}$ & Yes & pinnate & $9.47 \pm 3.85$ & $4.33 \pm 1.52$ \\
\hline Inga pavoniana & $\begin{array}{c}\text { Caesalpinioideae } \\
\text { (Mimosoideae) }\end{array}$ & Yes & pinnate & $12.77 \pm 4.68$ & $6.25 \pm 2.89$ \\
\hline Inga pezizifera & $\begin{array}{c}\text { Caesalpinioideae } \\
\text { (Mimosoideae) }\end{array}$ & Yes & pinnate & $10.54 \pm 3.95$ & $4.58 \pm 1.09$ \\
\hline Inga pilosula & $\begin{array}{c}\text { Caesalpinioideae } \\
\text { (Mimosoideae) }\end{array}$ & Yes & pinnate & $10.2 \pm 3.75$ & $4.51 \pm 1.37$ \\
\hline Inga pleiogyna & $\begin{array}{c}\text { Caesalpinioideae } \\
\text { (Mimosoideae) }\end{array}$ & Yes & pinnate & $15.81 \pm 4.8$ & $7.06 \pm 2.06$ \\
\hline Inga portobellensis & $\begin{array}{c}\text { Caesalpinioideae } \\
\text { (Mimosoideae) }\end{array}$ & Yes & pinnate & & \\
\hline Inga punctata & $\begin{array}{c}\text { Caesalpinioideae } \\
\text { (Mimosoideae) }\end{array}$ & Yes & pinnate & $10.84 \pm 4.49$ & $5.26 \pm 2.26$ \\
\hline Inga rubiginosa & $\begin{array}{c}\text { Caesalpinioideae } \\
\text { (Mimosoideae) }\end{array}$ & Yes & pinnate & $9.36 \pm 3.3$ & $4.91 \pm 1.84$ \\
\hline Inga ruiziana & $\begin{array}{c}\text { Caesalpinioideae } \\
\text { (Mimosoideae) }\end{array}$ & Yes & pinnate & $15.63 \pm 5.45$ & $7.29 \pm 2.4$ \\
\hline Inga sapindioides & $\begin{array}{c}\text { Caesalpinioideae } \\
\text { (Mimosoideae) }\end{array}$ & Yes & pinnate & & \\
\hline Inga sertulifera & $\begin{array}{c}\text { Caesalpinioideae } \\
\text { (Mimosoideae) }\end{array}$ & Yes & pinnate & $10.58 \pm 3.67$ & $4.63 \pm 1.91$ \\
\hline
\end{tabular}




\begin{tabular}{|c|c|c|c|c|c|}
\hline Species & Subfamily & $\begin{array}{l}\text { Potential } \\
\text { to fix } \mathrm{N}_{2}\end{array}$ & Leaf type & $\begin{array}{l}\text { Leaflet } \\
\text { length }\end{array}$ & $\begin{array}{l}\text { Leaflet } \\
\text { width }\end{array}$ \\
\hline Inga spectabilis & $\begin{array}{c}\text { Caesalpinioideae } \\
\text { (Mimosoideae) }\end{array}$ & Yes & pinnate & $11.68 \pm 4.77$ & $5.99 \pm 2.9$ \\
\hline Inga stipularis & $\begin{array}{c}\text { Caesalpinioideae } \\
\text { (Mimosoideae) }\end{array}$ & Yes & pinnate & $11.96 \pm 2.58$ & $5.32 \pm 1.56$ \\
\hline Inga tenuis & $\begin{array}{c}\text { Caesalpinioideae } \\
\text { (Mimosoideae) }\end{array}$ & Yes & pinnate & $2.17 \pm 0.63$ & $0.9 \pm 0.18$ \\
\hline Inga thibaudiana & $\begin{array}{c}\text { Caesalpinioideae } \\
\text { (Mimosoideae) }\end{array}$ & Yes & pinnate & $9.06 \pm 2.86$ & $4.48 \pm 1.55$ \\
\hline Inga tonduzii & $\begin{array}{c}\text { Caesalpinioideae } \\
\text { (Mimosoideae) }\end{array}$ & Yes & pinnate & $10.54 \pm 4.34$ & $5.62 \pm 1.92$ \\
\hline Inga umbellifera & $\begin{array}{c}\text { Caesalpinioideae } \\
\text { (Mimosoideae) }\end{array}$ & Yes & pinnate & $7.6 \pm 2.57$ & $2.3 \pm 0.65$ \\
\hline Inga venusta & $\begin{array}{c}\text { Caesalpinioideae } \\
\text { (Mimosoideae) }\end{array}$ & Yes & pinnate & $11.65 \pm 4.49$ & $4.95 \pm 2.65$ \\
\hline Inga vera & $\begin{array}{c}\text { Caesalpinioideae } \\
\text { (Mimosoideae) }\end{array}$ & Yes & pinnate & $9.36 \pm 4.12$ & $3.69 \pm 1.62$ \\
\hline $\begin{array}{l}\text { Leptolobium } \\
\text { panamense }\end{array}$ & Papilionoideae & Yes & pinnate & $4.85 \pm 1.5$ & $2.1 \pm 0.43$ \\
\hline Leucaena lanceolata & $\begin{array}{c}\text { Caesalpinioideae } \\
\text { (Mimosoideae) }\end{array}$ & Yes & bipinnate & $2.72 \pm 1.08$ & $1.14 \pm 0.4$ \\
\hline Leucaena leucocephala & $\begin{array}{c}\text { Caesalpinioideae } \\
\text { (Mimosoideae) }\end{array}$ & Yes & bipinnate & $0.86 \pm 0.2$ & $0.19 \pm 0.04$ \\
\hline $\begin{array}{l}\text { Lonchocarpus } \\
\text { acuminatus }\end{array}$ & Papilionoideae & Yes & pinnate & $5.74 \pm 1.22$ & $3 \pm 0.75$ \\
\hline $\begin{array}{l}\text { Lonchocarpus } \\
\text { campestris }\end{array}$ & Papilionoideae & Yes & pinnate & $3.66 \pm 1.58$ & $1.56 \pm 0.44$ \\
\hline Lonchocarpus castilloi & Papilionoideae & Yes & pinnate & $4.57 \pm 1.13$ & $1.21 \pm 0.18$ \\
\hline $\begin{array}{l}\text { Lonchocarpus } \\
\text { costaricensis }\end{array}$ & Papilionoideae & Yes & pinnate & $8.56 \pm 3.86$ & $5.27 \pm 2.14$ \\
\hline Lonchocarpus felipei & Papilionoideae & Yes & pinnate & $10.13 \pm 4.51$ & $5.9 \pm 2.76$ \\
\hline $\begin{array}{l}\text { Lonchocarpus } \\
\text { ferrugineus }\end{array}$ & Papilionoideae & Yes & pinnate & $20.37 \pm 4.4$ & $8.84 \pm 2.28$ \\
\hline $\begin{array}{l}\text { Lonchocarpus } \\
\text { guatemalensis }\end{array}$ & Papilionoideae & Yes & pinnate & $6.99 \pm 2.55$ & $3.15 \pm 1.19$ \\
\hline
\end{tabular}




\begin{tabular}{|c|c|c|c|c|c|}
\hline Species & Subfamily & $\begin{array}{l}\text { Potential } \\
\text { to fix } \mathrm{N}_{2}\end{array}$ & Leaf type & $\begin{array}{l}\text { Leaflet } \\
\text { length }\end{array}$ & $\begin{array}{l}\text { Leaflet } \\
\text { width }\end{array}$ \\
\hline $\begin{array}{l}\text { Lonchocarpus } \\
\text { heptaphyllus }\end{array}$ & Papilionoideae & Yes & pinnate & $9.68 \pm 2.74$ & $4.31 \pm 1.46$ \\
\hline $\begin{array}{l}\text { Lonchocarpus } \\
\text { hondurensis }\end{array}$ & Papilionoideae & Yes & pinnate & $6.36 \pm 2.29$ & $2.91 \pm 1.06$ \\
\hline Lonchocarpus hughesii & Papilionoideae & Yes & pinnate & $1.32 \pm 0.25$ & $0.58 \pm 0.06$ \\
\hline $\begin{array}{l}\text { Lonchocarpus } \\
\text { lanceolatus }\end{array}$ & Papilionoideae & Yes & pinnate & $4.85 \pm 0.84$ & $1.92 \pm 0.31$ \\
\hline $\begin{array}{l}\text { Lonchocarpus } \\
\text { macrophyllus }\end{array}$ & Papilionoideae & Yes & pinnate & & \\
\hline $\begin{array}{l}\text { Lonchocarpus } \\
\text { minimiflorus }\end{array}$ & Papilionoideae & Yes & pinnate & $3.48 \pm 1.69$ & $1.65 \pm 0.77$ \\
\hline $\begin{array}{l}\text { Lonchocarpus } \\
\text { montanus }\end{array}$ & Papilionoideae & Yes & pinnate & & \\
\hline Lonchocarpus mutans & Papilionoideae & Yes & pinnate & $5.44 \pm 0.87$ & $2.44 \pm 0.44$ \\
\hline $\begin{array}{l}\text { Lonchocarpus } \\
\text { parviflorus }\end{array}$ & Papilionoideae & Yes & pinnate & $3.45 \pm 1.51$ & $1.68 \pm 0.71$ \\
\hline $\begin{array}{l}\text { Lonchocarpus } \\
\text { peninsularis }\end{array}$ & Papilionoideae & Yes & pinnate & $5.74 \pm 1.22$ & $3 \pm 0.75$ \\
\hline $\begin{array}{l}\text { Lonchocarpus } \\
\text { phaseolifolius }\end{array}$ & Papilionoideae & Yes & pinnate & $6.46 \pm 4.2$ & $4.49 \pm 2.66$ \\
\hline Lonchocarpus rugosus & Papilionoideae & Yes & pinnate & $4.14 \pm 1.01$ & $1.6 \pm 0.23$ \\
\hline $\begin{array}{l}\text { Lonchocarpus } \\
\text { salvadorensis }\end{array}$ & Papilionoideae & Yes & pinnate & $7.21 \pm 1.39$ & $3.19 \pm 0.71$ \\
\hline Lonchocarpus sericeus & Papilionoideae & Yes & pinnate & $7.94 \pm 2.75$ & $3.95 \pm 0.97$ \\
\hline $\begin{array}{l}\text { Lonchocarpus } \\
\text { torresiorum }\end{array}$ & Papilionoideae & Yes & pinnate & & \\
\hline Lonchocarpus velutinus & Papilionoideae & Yes & pinnate & $10.56 \pm 4.32$ & $4.32 \pm 1.9$ \\
\hline Lonchocarpus xuul & Papilionoideae & Yes & pinnate & $4.99 \pm 1.08$ & $2.28 \pm 0.4$ \\
\hline $\begin{array}{l}\text { Lonchocarpus } \\
\text { yucatanensis }\end{array}$ & Papilionoideae & Yes & pinnate & $4.15 \pm 1.4$ & $1.88 \pm 0.46$ \\
\hline $\begin{array}{l}\text { Luetzelburgia andrade- } \\
\text { limae }\end{array}$ & Papilionoideae & No & pinnate & & \\
\hline Lysiloma acapulcense & $\begin{array}{c}\text { Caesalpinioideae } \\
\text { (Mimosoideae) }\end{array}$ & Yes & bipinnate & $0.33 \pm 0.09$ & $0.09 \pm 0.01$ \\
\hline
\end{tabular}




\begin{tabular}{|c|c|c|c|c|c|}
\hline Species & Subfamily & $\begin{array}{l}\text { Potential } \\
\text { to fix } \mathrm{N}_{2}\end{array}$ & Leaf type & $\begin{array}{l}\text { Leaflet } \\
\text { length }\end{array}$ & $\begin{array}{l}\text { Leaflet } \\
\text { width }\end{array}$ \\
\hline Lysiloma auritum & $\begin{array}{c}\text { Caesalpinioideae } \\
\text { (Mimosoideae) }\end{array}$ & Yes & bipinnate & $0.2 \pm 0.13$ & $0.35 \pm 0.69$ \\
\hline Lysiloma divaricatum & $\begin{array}{c}\text { Caesalpinioideae } \\
\text { (Mimosoideae) }\end{array}$ & Yes & bipinnate & $0.34 \pm 0.21$ & $0.13 \pm 0.09$ \\
\hline Lysiloma latisiliquum & $\begin{array}{c}\text { Caesalpinioideae } \\
\text { (Mimosoideae) }\end{array}$ & Yes & bipinnate & $1.75 \pm 2.57$ & $1.03 \pm 1.46$ \\
\hline Lysiloma microphyllum & $\begin{array}{l}\text { Caesalpinioideae } \\
\text { (Mimosoideae) }\end{array}$ & Yes & bipinnate & $0.3 \pm 0.08$ & $0.12 \pm 0.03$ \\
\hline $\begin{array}{l}\text { Machaerium } \\
\text { acutifolium }\end{array}$ & Papilionoideae & Yes & pinnate & $5.93 \pm 0.84$ & $2.75 \pm 0.31$ \\
\hline $\begin{array}{l}\text { Machaerium } \\
\text { biovulatum }\end{array}$ & Papilionoideae & Yes & pinnate & $4.02 \pm 0.87$ & $2.24 \pm 0.35$ \\
\hline $\begin{array}{l}\text { Machaerium } \\
\text { brasiliense }\end{array}$ & Papilionoideae & Yes & pinnate & $4.43 \pm 1.16$ & $1.69 \pm 0.26$ \\
\hline Machaerium hirtum & Papilionoideae & Yes & pinnate & $1.47 \pm 0.28$ & $0.37 \pm 0.06$ \\
\hline $\begin{array}{l}\text { Machaerium } \\
\text { hoehneanum }\end{array}$ & Papilionoideae & Yes & pinnate & $7.26 \pm 1.86$ & $2.57 \pm 0.3$ \\
\hline Machaerium nyctitans & Papilionoideae & Yes & pinnate & $2.18 \pm 0.51$ & $0.87 \pm 0.31$ \\
\hline Machaerium pittieri & Papilionoideae & Yes & pinnate & & \\
\hline $\begin{array}{l}\text { Machaerium } \\
\text { scleroxylon }\end{array}$ & Papilionoideae & Yes & pinnate & $1.62 \pm 0.63$ & $0.64 \pm 0.19$ \\
\hline Machaerium stipitatum & Papilionoideae & Yes & pinnate & $3.03 \pm 1.09$ & $1.21 \pm 0.56$ \\
\hline Machaerium vestitum & Papilionoideae & Yes & pinnate & $3.85 \pm 0.77$ & $1.6 \pm 0.22$ \\
\hline Machaerium villosum & Papilionoideae & Yes & pinnate & $5.14 \pm 1.26$ & $2.14 \pm 0.44$ \\
\hline $\begin{array}{l}\text { Macrolobium } \\
\text { costaricense }\end{array}$ & $\begin{array}{c}\text { Detarioideae } \\
\text { (Caesalpinioideae) }\end{array}$ & No & pinnate & & \\
\hline Macrolobium latifolium & $\begin{array}{c}\text { Detarioideae } \\
\text { (Caesalpinioideae) }\end{array}$ & No & pinnate & $9.42 \pm 5.19$ & $4 \pm 2.26$ \\
\hline Macrolobium limbatum & $\begin{array}{c}\text { Detarioideae } \\
\text { (Caesalpinioideae) }\end{array}$ & No & pinnate & $14.01 \pm 1.2$ & $5.69 \pm 0.52$ \\
\hline Mariosousa centralis & $\begin{array}{c}\text { Caesalpinioideae } \\
\text { (Mimosoideae) }\end{array}$ & unknown & bipinnate & $0.25 \pm 0.1$ & $0.1 \pm 0.03$ \\
\hline $\begin{array}{l}\text { Mariosousa } \\
\text { usumacintensis }\end{array}$ & $\begin{array}{l}\text { Caesalpinioideae } \\
\text { (Mimosoideae) }\end{array}$ & unknown & bipinnate & $0.39 \pm 0.11$ & $0.12 \pm 0.03$ \\
\hline
\end{tabular}




\begin{tabular}{|c|c|c|c|c|c|}
\hline Species & Subfamily & $\begin{array}{l}\text { Potential } \\
\text { to fix } \mathrm{N}_{2}\end{array}$ & Leaf type & $\begin{array}{l}\text { Leaflet } \\
\text { length }\end{array}$ & $\begin{array}{l}\text { Leaflet } \\
\text { width }\end{array}$ \\
\hline $\begin{array}{l}\text { Marmaroxylon } \\
\text { racemosum }\end{array}$ & $\begin{array}{c}\text { Caesalpinioideae } \\
\text { (Mimosoideae) }\end{array}$ & No & bipinnate & $1.04 \pm 0.22$ & $0.44 \pm 0.09$ \\
\hline $\begin{array}{l}\text { Mimosa acantholoba } \\
\text { var eurycarpa }\end{array}$ & $\begin{array}{c}\text { Caesalpinioideae } \\
\text { (Mimosoideae) }\end{array}$ & Yes & bipinnate & $0.56 \pm 0.22$ & $0.14 \pm 0.09$ \\
\hline Mimosa arenosa & $\begin{array}{c}\text { Caesalpinioideae } \\
\text { (Mimosoideae) }\end{array}$ & Yes & bipinnate & $0.38 \pm 0.13$ & $0.09 \pm 0.03$ \\
\hline Mimosa bahamensis & $\begin{array}{c}\text { Caesalpinioideae } \\
\text { (Mimosoideae) }\end{array}$ & Yes & bipinnate & $0.32 \pm 0.09$ & $0.21 \pm 0.07$ \\
\hline Mimosa tenuiflora & $\begin{array}{c}\text { Caesalpinioideae } \\
\text { (Mimosoideae) }\end{array}$ & Yes & bipinnate & $0.5 \pm 0.16$ & $0.12 \pm 0.02$ \\
\hline $\begin{array}{l}\text { Moldenhauera } \\
\text { blanchetiana }\end{array}$ & Caesalpinioideae & Yes & pinnate & & \\
\hline $\begin{array}{l}\text { Myrospermum } \\
\text { frutescens }\end{array}$ & Papilionoideae & No & pinnate & & \\
\hline Myroxylon peruiferum & Papilionoideae & No & pinnate & $5.15 \pm 0.98$ & $2.45 \pm 0.12$ \\
\hline Ormosia amazonica & Papilionoideae & Yes & pinnate & $15.47 \pm 4.41$ & $7.14 \pm 1.59$ \\
\hline Ormosia coccinea & Papilionoideae & Yes & pinnate & $14.5 \pm 4.43$ & $7.35 \pm 0.93$ \\
\hline Ormosia discolor & Papilionoideae & Yes & pinnate & $13.02 \pm 3.65$ & $5.12 \pm 0.82$ \\
\hline Ormosia flava & Papilionoideae & Yes & pinnate & $11.01 \pm 3.32$ & $4.4 \pm 1.48$ \\
\hline Ormosia grossa & Papilionoideae & Yes & pinnate & $12.48 \pm 3.91$ & $7.6 \pm 1.72$ \\
\hline Ormosia isthmensis & Papilionoideae & Yes & pinnate & $10.92 \pm 2.89$ & $5.34 \pm 0.45$ \\
\hline Ormosia krugii & Papilionoideae & Yes & pinnate & $15.03 \pm 3.01$ & $7.53 \pm 1.29$ \\
\hline Ormosia macrocalyx & Papilionoideae & Yes & pinnate & $9.23 \pm 2.2$ & $4.47 \pm 0.84$ \\
\hline Ormosia macrophylla & Papilionoideae & Yes & pinnate & $11.88 \pm 2.73$ & $6.54 \pm 1.71$ \\
\hline Ormosia nobilis & Papilionoideae & Yes & pinnate & $16.91 \pm 2.77$ & $7.37 \pm 0.74$ \\
\hline Ormosia paraensis & Papilionoideae & Yes & pinnate & $6.02 \pm 0.99$ & $3.28 \pm 0.76$ \\
\hline Ormosia subsimplex & Papilionoideae & Yes & pinnate & $11.07 \pm 2.77$ & $4.29 \pm 0.74$ \\
\hline Ormosia velutina & Papilionoideae & Yes & pinnate & $8 \pm 2.24$ & $4.79 \pm 0.92$ \\
\hline Parkia bahiae & $\begin{array}{c}\text { Caesalpinioideae } \\
\text { (Mimosoideae) }\end{array}$ & No & bipinnate & $2.1 \pm 0.32$ & $0.49 \pm 0.06$ \\
\hline Parkia decussata & $\begin{array}{c}\text { Caesalpinioideae } \\
\text { (Mimosoideae) }\end{array}$ & No & bipinnate & $2.82 \pm 0.53$ & $0.73 \pm 0.15$ \\
\hline Parkia multijuga & $\begin{array}{c}\text { Caesalpinioideae } \\
\text { (Mimosoideae) }\end{array}$ & No & bipinnate & $0.87 \pm 0.13$ & $0.25 \pm 0.04$ \\
\hline
\end{tabular}




\begin{tabular}{|c|c|c|c|c|c|}
\hline Species & Subfamily & $\begin{array}{l}\text { Potential } \\
\text { to fix } \mathrm{N}_{2}\end{array}$ & Leaf type & $\begin{array}{l}\text { Leaflet } \\
\text { length }\end{array}$ & $\begin{array}{l}\text { Leaflet } \\
\text { width }\end{array}$ \\
\hline Parkia nitida & $\begin{array}{c}\text { Caesalpinioideae } \\
\text { (Mimosoideae) }\end{array}$ & No & bipinnate & $1.13 \pm 0.42$ & $0.31 \pm 0.09$ \\
\hline Parkia oppositifolia & $\begin{array}{c}\text { Caesalpinioideae } \\
\text { (Mimosoideae) }\end{array}$ & No & bipinnate & $0.83 \pm 0.29$ & $0.3 \pm 0.09$ \\
\hline Parkia pendula & $\begin{array}{c}\text { Caesalpinioideae } \\
\text { (Mimosoideae) }\end{array}$ & No & bipinnate & $0.33 \pm 0.07$ & $0.08 \pm 0.02$ \\
\hline Parkinsonia praecox & Caesalpinioideae & No & bipinnate & $0.65 \pm 0.16$ & $0.25 \pm 0.04$ \\
\hline Peltogyne pauciflora & $\begin{array}{c}\text { Detarioideae } \\
\text { (Caesalpinioideae) }\end{array}$ & No & pinnate & $5.64 \pm 1.73$ & $2.55 \pm 0.75$ \\
\hline Peltogyne purpurea & $\begin{array}{c}\text { Detarioideae } \\
\text { (Caesalpinioideae) }\end{array}$ & No & pinnate & $5.59 \pm 0.24$ & $2.33 \pm 0.27$ \\
\hline Peltogyne venosa & $\begin{array}{c}\text { Detarioideae } \\
\text { (Caesalpinioideae) }\end{array}$ & No & pinnate & $11.18 \pm 1.57$ & $4.43 \pm 0.56$ \\
\hline Peltophorum dubium & Caesalpinioideae & No & bipinnate & $0.67 \pm 0.16$ & $0.24 \pm 0.07$ \\
\hline $\begin{array}{l}\text { Pentaclethra } \\
\text { macroloba }\end{array}$ & $\begin{array}{c}\text { Caesalpinioideae } \\
\text { (Mimosoideae) }\end{array}$ & Yes & bipinnate & $0.57 \pm 0.2$ & $0.22 \pm 0.17$ \\
\hline Piptadenia constricta & $\begin{array}{c}\text { Caesalpinioideae } \\
\text { (Mimosoideae) }\end{array}$ & Yes & bipinnate & $0.91 \pm 0.3$ & $0.49 \pm 0.17$ \\
\hline $\begin{array}{l}\text { Piptadenia } \\
\text { gonoacantha }\end{array}$ & $\begin{array}{c}\text { Caesalpinioideae } \\
\text { (Mimosoideae) }\end{array}$ & Yes & bipinnate & $0.59 \pm 0.19$ & $0.13 \pm 0.03$ \\
\hline $\begin{array}{l}\text { Piptadenia } \\
\text { moniliformis }\end{array}$ & $\begin{array}{c}\text { Caesalpinioideae } \\
\text { (Mimosoideae) }\end{array}$ & Yes & bipinnate & $1.21 \pm 0.38$ & $0.53 \pm 0.13$ \\
\hline Piptadenia obliqua & $\begin{array}{c}\text { Caesalpinioideae } \\
\text { (Mimosoideae) }\end{array}$ & Yes & bipinnate & $1.38 \pm 0.37$ & $0.87 \pm 0.23$ \\
\hline Piptadenia paniculata & $\begin{array}{c}\text { Caesalpinioideae } \\
\text { (Mimosoideae) }\end{array}$ & Yes & bipinnate & $2.95 \pm 0.88$ & $1.27 \pm 0.35$ \\
\hline Piptadenia stipulacea & $\begin{array}{c}\text { Caesalpinioideae } \\
\text { (Mimosoideae) }\end{array}$ & Yes & bipinnate & $0.54 \pm 0.16$ & $0.09 \pm 0.02$ \\
\hline Piptadenia viridiflora & $\begin{array}{c}\text { Caesalpinioideae } \\
\text { (Mimosoideae) }\end{array}$ & Yes & bipinnate & $0.5 \pm 0.1$ & $0.13 \pm 0.03$ \\
\hline Piscidia carthagenensis & Papilionoideae & Yes & pinnate & $6.16 \pm 1.29$ & $3.59 \pm 1.44$ \\
\hline Piscidia piscipula & Papilionoideae & Yes & pinnate & $7.6 \pm 1.64$ & $4.73 \pm 1.46$ \\
\hline $\begin{array}{l}\text { Pithecellobium } \\
\text { arboreum }\end{array}$ & $\begin{array}{c}\text { Caesalpinioideae } \\
\text { (Mimosoideae) }\end{array}$ & Yes & bipinnate & $0.94 \pm 0.31$ & $0.45 \pm 0.3$ \\
\hline
\end{tabular}




\begin{tabular}{|c|c|c|c|c|c|}
\hline Species & Subfamily & $\begin{array}{l}\text { Potential } \\
\text { to fix } \mathrm{N}_{2}\end{array}$ & Leaf type & $\begin{array}{l}\text { Leaflet } \\
\text { length }\end{array}$ & $\begin{array}{l}\text { Leaflet } \\
\text { width }\end{array}$ \\
\hline $\begin{array}{l}\text { Pithecellobium } \\
\text { bipinnatum }\end{array}$ & $\begin{array}{l}\text { Caesalpinioideae } \\
\text { (Mimosoideae) }\end{array}$ & Yes & bipinnate & $3.14 \pm 0.82$ & $1.5 \pm 0.31$ \\
\hline Pithecellobium dulce & $\begin{array}{c}\text { Caesalpinioideae } \\
\text { (Mimosoideae) }\end{array}$ & Yes & bipinnate & $2.68 \pm 0.78$ & $1.28 \pm 0.33$ \\
\hline $\begin{array}{l}\text { Pithecellobium } \\
\text { hymenaeifolium }\end{array}$ & $\begin{array}{c}\text { Caesalpinioideae } \\
\text { (Mimosoideae) }\end{array}$ & Yes & bipinnate & $6.97 \pm 2.41$ & $3.44 \pm 1.43$ \\
\hline $\begin{array}{l}\text { Pithecellobium } \\
\text { lanceolatum }\end{array}$ & $\begin{array}{l}\text { Caesalpinioideae } \\
\text { (Mimosoideae) }\end{array}$ & Yes & bipinnate & $4.7 \pm 0.85$ & $1.55 \pm 0.35$ \\
\hline Plathymenia reticulata & $\begin{array}{c}\text { Caesalpinioideae } \\
\text { (Mimosoideae) }\end{array}$ & Yes & bipinnate & $0.94 \pm 0.11$ & $0.41 \pm 0.06$ \\
\hline Platycyamus regnellii & Papilionoideae & Yes & pinnate & $15.34 \pm 3.23$ & $15.67 \pm 4.88$ \\
\hline Platymiscium blanchetii & Papilionoideae & Yes & pinnate & & \\
\hline Platymiscium curuense & Papilionoideae & Yes & pinnate & $10.76 \pm 3.02$ & $4.75 \pm 1.08$ \\
\hline $\begin{array}{l}\text { Platymiscium } \\
\text { dimorphandrum }\end{array}$ & Papilionoideae & Yes & pinnate & $6.29 \pm 1.45$ & $2.68 \pm 0.67$ \\
\hline $\begin{array}{l}\text { Platymiscium } \\
\text { lasiocarpum }\end{array}$ & Papilionoideae & Yes & pinnate & & \\
\hline $\begin{array}{l}\text { Platymiscium } \\
\text { parviflorum }\end{array}$ & Papilionoideae & Yes & pinnate & $8.09 \pm 2.24$ & $6.88 \pm 2.86$ \\
\hline Platymiscium pinnatum & Papilionoideae & Yes & pinnate & $6.53 \pm 1.97$ & $3.55 \pm 0.83$ \\
\hline $\begin{array}{l}\text { Platymiscium } \\
\text { speciosum }\end{array}$ & Papilionoideae & Yes & pinnate & $10.01 \pm 3.58$ & $4.67 \pm 1.33$ \\
\hline Platymiscium ulei & Papilionoideae & Yes & pinnate & $6.06 \pm 2.05$ & $2.7 \pm 0.9$ \\
\hline $\begin{array}{l}\text { Platymiscium } \\
\text { yucatanum }\end{array}$ & Papilionoideae & Yes & pinnate & $3.27 \pm 0.93$ & $1.56 \pm 0.36$ \\
\hline Platypodium elegans & Papilionoideae & Yes & pinnate & $2.89 \pm 0.49$ & $1.14 \pm 0.2$ \\
\hline Poecilanthe effusa & Papilionoideae & Yes & pinnate & $8.85 \pm 1.9$ & $3.2 \pm 0.49$ \\
\hline Poeppigia procera & $\begin{array}{c}\text { Dialioideae } \\
\text { (Caesalpinioideae) }\end{array}$ & No & pinnate & $1.5 \pm 0.42$ & $0.39 \pm 0.1$ \\
\hline Poincianella pluviosa & Caesalpinioideae & No & bipinnate & $2.04 \pm 0.52$ & $1.51 \pm 0.3$ \\
\hline Prioria copaifera & $\begin{array}{c}\text { Detarioideae } \\
\text { (Caesalpinioideae) }\end{array}$ & No & pinnate & $9.72 \pm 2.25$ & $4.74 \pm 1.19$ \\
\hline $\begin{array}{l}\text { Pseudopiptadenia } \\
\text { contorta }\end{array}$ & $\begin{array}{c}\text { Caesalpinioideae } \\
\text { (Mimosoideae) }\end{array}$ & Yes & bipinnate & $0.25 \pm 0.09$ & $0.07 \pm 0.01$ \\
\hline
\end{tabular}




\begin{tabular}{|c|c|c|c|c|c|}
\hline Species & Subfamily & $\begin{array}{l}\text { Potential } \\
\text { to fix } \mathrm{N}_{2}\end{array}$ & Leaf type & $\begin{array}{l}\text { Leaflet } \\
\text { length }\end{array}$ & $\begin{array}{l}\text { Leaflet } \\
\text { width }\end{array}$ \\
\hline $\begin{array}{l}\text { Pseudosamanea } \\
\text { guachapele }\end{array}$ & $\begin{array}{l}\text { Caesalpinioideae } \\
\text { (Mimosoideae) }\end{array}$ & Yes & bipinnate & $2.91 \pm 1$ & $1.53 \pm 0.51$ \\
\hline $\begin{array}{l}\text { Pterocarpus } \\
\text { acapulcensis }\end{array}$ & Papilionoideae & Yes & pinnate & $5.7 \pm 2.17$ & $2.86 \pm 1.18$ \\
\hline Pterocarpus hayesii & Papilionoideae & Yes & pinnate & $11.12 \pm 2.94$ & $4.18 \pm 0.49$ \\
\hline $\begin{array}{l}\text { Pterocarpus } \\
\text { michelianus }\end{array}$ & Papilionoideae & Yes & pinnate & & \\
\hline Pterocarpus officinalis & Papilionoideae & Yes & pinnate & $8.9 \pm 1.41$ & $4.54 \pm 0.4$ \\
\hline $\begin{array}{l}\text { Pterocarpus } \\
\text { orbiculatus }\end{array}$ & Papilionoideae & Yes & pinnate & $8.53 \pm 0.81$ & $3.27 \pm 0.03$ \\
\hline Pterocarpus rohrii & Papilionoideae & Yes & pinnate & $9.61 \pm 1.71$ & $4.17 \pm 0.79$ \\
\hline Pterocarpus violaceus & Papilionoideae & Yes & pinnate & $6.15 \pm 1.74$ & $2.96 \pm 0.47$ \\
\hline Pterocarpus zehntneri & Papilionoideae & Yes & pinnate & & \\
\hline Pterodon abruptus & Papilionoideae & No & pinnate & $1.54 \pm 0.3$ & $1.07 \pm 0.17$ \\
\hline Pterogyne nitens & Caesalpinioideae & No & pinnate & $4.87 \pm 1.01$ & $2.06 \pm 0.41$ \\
\hline Samanea saman & $\begin{array}{l}\text { Caesalpinioideae } \\
\text { (Mimosoideae) }\end{array}$ & Yes & bipinnate & $3.42 \pm 1.5$ & $1.65 \pm 0.54$ \\
\hline $\begin{array}{l}\text { Schizolobium } \\
\text { amazonicum }\end{array}$ & Caesalpinioideae & No & bipinnate & $3.35 \pm 1.35$ & $2.26 \pm 0.87$ \\
\hline Schizolobium parahyba & Caesalpinioideae & No & bipinnate & $2.96 \pm 0.43$ & $0.78 \pm 0.1$ \\
\hline $\begin{array}{l}\text { Sclerolobium } \\
\text { costaricense }\end{array}$ & Caesalpinioideae & Yes & pinnate & $12.69 \pm 2.29$ & $5.53 \pm 0.88$ \\
\hline $\begin{array}{l}\text { Sclerolobium } \\
\text { densiflorum }\end{array}$ & Caesalpinioideae & Yes & pinnate & $8.54 \pm 2.25$ & $5.1 \pm 1.17$ \\
\hline $\begin{array}{l}\text { Sclerolobium } \\
\text { paniculatum }\end{array}$ & Caesalpinioideae & Yes & pinnate & $10.27 \pm 1.96$ & $3.94 \pm 0.92$ \\
\hline Sclerolobium paraense & Caesalpinioideae & Yes & pinnate & $12.27 \pm 2.9$ & $4.04 \pm 0.81$ \\
\hline Senegalia gaumeri & $\begin{array}{l}\text { Caesalpinioideae } \\
\text { (Mimosoideae) }\end{array}$ & Yes & bipinnate & $0.9 \pm 0.21$ & $0.33 \pm 0.06$ \\
\hline Senegalia langsdorffii & $\begin{array}{l}\text { Caesalpinioideae } \\
\text { (Mimosoideae) }\end{array}$ & Yes & bipinnate & $0.99 \pm 1.18$ & $0.84 \pm 1.76$ \\
\hline Senegalia loretensis & $\begin{array}{c}\text { Caesalpinioideae } \\
\text { (Mimosoideae) }\end{array}$ & unknown & bipinnate & $0.62 \pm 0.14$ & $0.14 \pm 0.04$ \\
\hline
\end{tabular}




\begin{tabular}{|c|c|c|c|c|c|}
\hline Species & Subfamily & $\begin{array}{l}\text { Potential } \\
\text { to fix } \mathrm{N}_{2}\end{array}$ & Leaf type & $\begin{array}{l}\text { Leaflet } \\
\text { length }\end{array}$ & $\begin{array}{l}\text { Leaflet } \\
\text { width }\end{array}$ \\
\hline Senegalia martii & $\begin{array}{c}\text { Caesalpinioideae } \\
\text { (Mimosoideae) }\end{array}$ & Yes & bipinnate & $0.34 \pm 0.07$ & $0.09 \pm 0.02$ \\
\hline Senegalia piauhiensis & $\begin{array}{c}\text { Caesalpinioideae } \\
\text { (Mimosoideae) }\end{array}$ & Yes & bipinnate & $0.88 \pm 0.16$ & $0.26 \pm 0.08$ \\
\hline Senegalia picachensis & $\begin{array}{c}\text { Caesalpinioideae } \\
\text { (Mimosoideae) }\end{array}$ & unknown & bipinnate & $0.8 \pm 0.14$ & $0.25 \pm 0.08$ \\
\hline Senegalia polyphylla & $\begin{array}{c}\text { Caesalpinioideae } \\
\text { (Mimosoideae) }\end{array}$ & No & bipinnate & & \\
\hline Senna acuruensis & Caesalpinioideae & No & pinnate & $1.36 \pm 0.2$ & $0.65 \pm 0.07$ \\
\hline Senna atomaria & Caesalpinioideae & No & pinnate & $3.73 \pm 1.81$ & $2.38 \pm 1.19$ \\
\hline Senna bacillaris & Caesalpinioideae & No & pinnate & $10.5 \pm 3.16$ & $4.6 \pm 0.87$ \\
\hline Senna fruticosa & Caesalpinioideae & No & pinnate & $7.89 \pm 2.1$ & $3.87 \pm 1.51$ \\
\hline Senna hayesiana & Caesalpinioideae & No & pinnate & $9.08 \pm 2.93$ & $4.08 \pm 0.97$ \\
\hline Senna multijuga & Caesalpinioideae & No & pinnate & $2.14 \pm 0.75$ & $0.6 \pm 0.22$ \\
\hline Senna papillosa & Caesalpinioideae & No & pinnate & $14.65 \pm 2.75$ & $6.13 \pm 0.83$ \\
\hline Senna racemosa & Caesalpinioideae & No & pinnate & $7.89 \pm 2.1$ & $3.87 \pm 1.51$ \\
\hline Senna spectabilis & Caesalpinioideae & No & pinnate & $5.04 \pm 1.09$ & $1.83 \pm 0.29$ \\
\hline Senna sylvestris & Caesalpinioideae & No & pinnate & $6.96 \pm 1.73$ & $2.47 \pm 0.48$ \\
\hline Senna undulata & Caesalpinioideae & No & pinnate & $6.11 \pm 1.79$ & $3.01 \pm 0.77$ \\
\hline Senna velutina & Caesalpinioideae & No & pinnate & $5.13 \pm 1.6$ & $2.1 \pm 0.65$ \\
\hline Senna villosa & Caesalpinioideae & No & pinnate & $3.97 \pm 0.89$ & $1.66 \pm 0.34$ \\
\hline $\begin{array}{l}\text { Stryphnodendron } \\
\text { barbatimam }\end{array}$ & $\begin{array}{c}\text { Caesalpinioideae } \\
\text { (Mimosoideae) }\end{array}$ & Yes & bipinnate & $1.6 \pm 0.17$ & $1.4 \pm 0.1$ \\
\hline $\begin{array}{l}\text { Stryphnodendron } \\
\text { guianense }\end{array}$ & $\begin{array}{c}\text { Caesalpinioideae } \\
\text { (Mimosoideae) }\end{array}$ & Yes & bipinnate & $0.85 \pm 0.27$ & $0.37 \pm 0.11$ \\
\hline $\begin{array}{l}\text { Stryphnodendron } \\
\text { microstachyum }\end{array}$ & $\begin{array}{c}\text { Caesalpinioideae } \\
\text { (Mimosoideae) }\end{array}$ & Yes & bipinnate & $2.18 \pm 0.61$ & $1.03 \pm 0.25$ \\
\hline $\begin{array}{l}\text { Stryphnodendron } \\
\text { occhionianum }\end{array}$ & $\begin{array}{c}\text { Caesalpinioideae } \\
\text { (Mimosoideae) }\end{array}$ & Yes & bipinnate & & \\
\hline $\begin{array}{l}\text { Stryphnodendron } \\
\text { pulcherrimum }\end{array}$ & $\begin{array}{c}\text { Caesalpinioideae } \\
\text { (Mimosoideae) }\end{array}$ & Yes & bipinnate & $0.6 \pm 0.17$ & $0.21 \pm 0.07$ \\
\hline $\begin{array}{l}\text { Stryphnodendron } \\
\text { purpureum }\end{array}$ & $\begin{array}{c}\text { Caesalpinioideae } \\
\text { (Mimosoideae) }\end{array}$ & Yes & bipinnate & & \\
\hline Swartzia apetala & Papilionoideae & Yes & pinnate & $6.34 \pm 1.16$ & $2.94 \pm 0.46$ \\
\hline
\end{tabular}




\begin{tabular}{|c|c|c|c|c|c|}
\hline Species & Subfamily & $\begin{array}{l}\text { Potential } \\
\text { to fix } \mathrm{N}_{2}\end{array}$ & Leaf type & $\begin{array}{l}\text { Leaflet } \\
\text { length }\end{array}$ & $\begin{array}{l}\text { Leaflet } \\
\text { width }\end{array}$ \\
\hline $\begin{array}{l}\text { Swartzia apetala var } \\
\text { apetala }\end{array}$ & Papilionoideae & Yes & pinnate & $7.06 \pm 2.23$ & $3.4 \pm 1.11$ \\
\hline Swartzia arborescens & Papilionoideae & Yes & pinnate & $3.89 \pm 1.71$ & $2.12 \pm 0.55$ \\
\hline Swartzia brachyrachis & Papilionoideae & Yes & pinnate & $5.6 \pm 1.88$ & $2.68 \pm 0.66$ \\
\hline Swartzia cubensis & Papilionoideae & Yes & pinnate & $6.45 \pm 1.4$ & $2.13 \pm 0.18$ \\
\hline Swartzia cuspidata & Papilionoideae & Yes & pinnate & $7.91 \pm 1.44$ & $3.55 \pm 0.85$ \\
\hline Swartzia flaemingii & Papilionoideae & Yes & pinnate & $3.65 \pm 1.32$ & $1.39 \pm 0.64$ \\
\hline Swartzia laurifolia & Papilionoideae & Yes & pinnate & $17.87 \pm 5.79$ & $6.69 \pm 2.61$ \\
\hline Swartzia laxiflora & Papilionoideae & Yes & pinnate & $7.12 \pm 2.05$ & $2.41 \pm 0.63$ \\
\hline Swartzia macrostachya & Papilionoideae & Yes & pinnate & $5.2 \pm 1.97$ & $2.83 \pm 1.01$ \\
\hline Swartzia multijuga & Papilionoideae & Yes & pinnate & $7.73 \pm 2.23$ & $3.12 \pm 0.42$ \\
\hline Swartzia myrtifolia & Papilionoideae & Yes & pinnate & $3.76 \pm 1.07$ & $1.53 \pm 0.44$ \\
\hline Swartzia nicaraguensis & Papilionoideae & Yes & pinnate & $7.9 \pm 1.7$ & $3.63 \pm 0.67$ \\
\hline Swartzia ochnacea & Papilionoideae & Yes & unifoliolate & $14.37 \pm 8.32$ & $5.48 \pm 2.64$ \\
\hline Swartzia panamensis & Papilionoideae & Yes & pinnate & $9.74 \pm 3.37$ & $3.24 \pm 0.63$ \\
\hline Swartzia polyphylla & Papilionoideae & Yes & pinnate & $10.52 \pm 4.36$ & $3.83 \pm 1.61$ \\
\hline Swartzia reticulata & Papilionoideae & Yes & pinnate & $16.01 \pm 3.89$ & $8.47 \pm 1.44$ \\
\hline Swartzia schomburgkii & Papilionoideae & Yes & pinnate & $12.33 \pm 2.36$ & $5.24 \pm 0.91$ \\
\hline Swartzia simplex & Papilionoideae & Yes & unifoliolate & & \\
\hline $\begin{array}{l}\text { Swartzia simplex var } \\
\text { continentalis }\end{array}$ & Papilionoideae & Yes & unifoliolate & $9.41 \pm 2.71$ & $4.14 \pm 1.25$ \\
\hline $\begin{array}{l}\text { Swartzia simplex var } \\
\text { grandiflora }\end{array}$ & Papilionoideae & Yes & unifoliolate & $5.5 \pm 1.87$ & $2.75 \pm 0.8$ \\
\hline Swartzia tessmannii & Papilionoideae & Yes & pinnate & $7.75 \pm 2.67$ & $4.03 \pm 0.67$ \\
\hline Sweetia fruticosa & Papilionoideae & No & pinnate & $2.57 \pm 0.76$ & $1.27 \pm 0.2$ \\
\hline Tabaroa caatingicola & Papilionoideae & Yes & pinnate & $3.94 \pm 0.79$ & $2.21 \pm 0.27$ \\
\hline Tachigali cenepensis & Caesalpinioideae & Yes & pinnate & $18.63 \pm 6.15$ & $9.03 \pm 1.3$ \\
\hline Tachigali chrysaloides & Caesalpinioideae & Yes & pinnate & $11.12 \pm 3.7$ & $4.32 \pm 1.31$ \\
\hline Tachigali macbridei & Caesalpinioideae & Yes & pinnate & $10.5 \pm 3.68$ & $3.87 \pm 1.14$ \\
\hline $\begin{array}{l}\text { Tachigali } \\
\text { myrmecophila }\end{array}$ & Caesalpinioideae & Yes & pinnate & $9.64 \pm 3.01$ & $3.8 \pm 1.17$ \\
\hline Tachigali paniculata & Caesalpinioideae & Yes & pinnate & $7.93 \pm 1.88$ & $3.63 \pm 1.22$ \\
\hline Tachigali polyphylla & Caesalpinioideae & Yes & pinnate & $6.34 \pm 3.36$ & $2.03 \pm 1.2$ \\
\hline Tachigali vasquezii & Caesalpinioideae & Yes & pinnate & & \\
\hline Tachigali versicolor & Caesalpinioideae & Yes & pinnate & $15.4 \pm 4.9$ & $5.07 \pm 1.11$ \\
\hline
\end{tabular}




\begin{tabular}{|c|c|c|c|c|c|}
\hline Species & Subfamily & $\begin{array}{l}\text { Potential } \\
\text { to fix } \mathrm{N}_{2}\end{array}$ & Leaf type & $\begin{array}{l}\text { Leaflet } \\
\text { length }\end{array}$ & $\begin{array}{c}\text { Leaflet } \\
\text { width }\end{array}$ \\
\hline Vachellia allenii & $\begin{array}{c}\text { Caesalpinioideae } \\
\text { (Mimosoideae) }\end{array}$ & Yes & bipinnate & $0.7 \pm 0.07$ & $0.18 \pm 0.03$ \\
\hline Vachellia campechiana & $\begin{array}{c}\text { Caesalpinioideae } \\
\text { (Mimosoideae) }\end{array}$ & Yes & bipinnate & $0.11 \pm 0.01$ & $0.04 \pm 0.01$ \\
\hline Vachellia collinsii & $\begin{array}{c}\text { Caesalpinioideae } \\
\text { (Mimosoideae) }\end{array}$ & Yes & bipinnate & $0.69 \pm 0.15$ & $0.16 \pm 0.02$ \\
\hline Vachellia cornigera & $\begin{array}{c}\text { Caesalpinioideae } \\
\text { (Mimosoideae) }\end{array}$ & Yes & bipinnate & $0.62 \pm 0.12$ & $0.17 \pm 0.04$ \\
\hline Vachellia farnesiana & $\begin{array}{c}\text { Caesalpinioideae } \\
\text { (Mimosoideae) }\end{array}$ & Yes & bipinnate & $0.3 \pm 0.05$ & $0.08 \pm 0.02$ \\
\hline Vachellia macracantha & $\begin{array}{c}\text { Caesalpinioideae } \\
\text { (Mimosoideae) }\end{array}$ & Yes & bipinnate & $0.22 \pm 0.06$ & $0.06 \pm 0.01$ \\
\hline Vatairea erythrocarpa & Papilionoideae & No & pinnate & $10.13 \pm 3.21$ & $4.69 \pm 1.63$ \\
\hline Vatairea lundellii & Papilionoideae & No & pinnate & $5.56 \pm 1.88$ & $2.29 \pm 0.58$ \\
\hline Vataireopsis glaziovii & Papilionoideae & No & pinnate & & \\
\hline Vataireopsis iglesiasii & Papilionoideae & No & pinnate & $7.12 \pm 8.5$ & $6.67 \pm 7.42$ \\
\hline Zapoteca formosa & $\begin{array}{c}\text { Caesalpinioideae } \\
\text { (Mimosoideae) }\end{array}$ & Yes & bipinnate & $1.81 \pm 1.15$ & $0.98 \pm 0.6$ \\
\hline Zollernia latifolia & Papilionoideae & No & unifoliolate & $9.47 \pm 2.47$ & $9.36 \pm 2.11$ \\
\hline Zollernia paraensis & Papilionoideae & No & unifoliolate & $10.2 \pm 2.08$ & $3.69 \pm 0.68$ \\
\hline Zygia cognata & $\begin{array}{c}\text { Caesalpinioideae } \\
\text { (Mimosoideae) }\end{array}$ & Yes & pinnate & $7.71 \pm 3.08$ & $2.78 \pm 1.16$ \\
\hline Zygia gigantifoliola & $\begin{array}{c}\text { Caesalpinioideae } \\
\text { (Mimosoideae) }\end{array}$ & Yes & pinnate & $17.03 \pm 6.02$ & $5.57 \pm 2.4$ \\
\hline Zygia racemosa & $\begin{array}{c}\text { Caesalpinioideae } \\
\text { (Mimosoideae) }\end{array}$ & Yes & bipinnate & $0.74 \pm 0.06$ & $0.24 \pm 0.02$ \\
\hline
\end{tabular}





\section{Supplementary Table 4 | Comparison of linear mixed models addressing the effects}

of stand age and three climatic variables on legume relative abundance in Neotropical secondary forests.

Linear mixed models were run for relative basal area of all legume species with stand age and one of three climatic variables (mean annual rainfall, rainfall seasonality "RS", and climatic water deficit "CWD"). Random site intercepts account for between-site variation in initial legume basal area, and random slopes for the variation of the effect of stand age on legume basal area among sites. Standardized coefficients and $F$-values of each predictor and their interaction in linear mixed effects models are shown $\left(\left({ }^{\dagger} P<0.05\right.\right.$; ${ }^{*} P \leq$ $\left.0.01 ;{ }^{* *} P \leq 0.001 ;{ }^{* * *} P \leq 0.001\right)$. The standardized regression coefficients compare the effect of the independent variables on the dependent variable. Values of marginal $\left(R^{2}\right.$ $(\mathrm{m}))$ and conditional $\left(R^{2}(\mathrm{c})\right) R^{2}$ indicate the proportion of the variance explained by the fixed predictors of the model, and the fit of the whole model with fixed and random factors, respectively. Change in AIC for small sample sizes ( $\triangle \mathrm{AICc})$ compared with the best model (A) and $R^{2}$ are shown. For all models $N=41$ chronosequence sites (one site was excluded because no climatic data were available).

\begin{tabular}{lllllll}
\hline Dependent variable & Parameter & $\begin{array}{l}\text { Standardized } \\
\text { coefficients }\end{array}$ & F-value & $\boldsymbol{R}^{2}(\mathbf{m})$ & $\boldsymbol{R}^{2}(\mathbf{c})$ & $\Delta$ AICc \\
\hline A. Legume relative basal area & & -0.61 & $21.15^{* *}$ & 17.18 & 62.14 & 0.00 \\
Stand age & -0.95 & $16.23^{* *}$ & & & \\
Rainfall & 0.62 & $12.01^{*}$ & & & \\
Stand age $\times$ Rainfall & & & & \\
& & & 10.45 & 48.89 & 13.93 \\
B. Legume relative basal area & 0.22 & $2.08^{\text {n.s. }}$ & & & \\
Stand age & 0.61 & $9.81^{* *}$ & & & \\
RS & -0.49 & $6.05^{*}$ & & & \\
Stand age $\times$ RS & & & & & \\
C. Legume relative basal area & & & & \\
Stand age & 0.11 & $1.56^{\text {n.s. }}$ & & & \\
CWD & -0.89 & $22.51^{* * *}$ & & & \\
Stand age $\times$ CWD & 0.59 & $13.33^{* * *}$ & & & \\
\hline
\end{tabular}




\section{Supplementary Table 5 | Effects of stand age and rainfall on the abundance of non- fixing legumes in Neotropical secondary forests.}

Linear mixed models were run for absolute and relative basal area of non-fixing legume species (A and B respectively). Random site intercepts account for between-site variation in initial legume basal area, and random slopes for the variation of the effect of stand age on legume basal area among sites. Standardized coefficients and $F$-values of each predictor and their interaction in linear mixed effects models are shown $\left({ }^{\dagger} P<0.05 ;{ }^{*} P \leq\right.$ $\left.0.01 ;{ }^{* *} P \leq 0.001 ;{ }^{* * *} P \leq 0.001\right)$. The standardized regression coefficients compare the effect of the independent variables on the dependent variable. Values of marginal $\left(R^{2}\right.$ $(\mathrm{m}))$ and conditional $\left(R^{2}(\mathrm{c})\right) R^{2}$ indicate the proportion of the variance explained by the fixed predictors of the model, and the fit of the whole model with fixed and random factors, respectively. $N=42$ chronosequence sites.

\begin{tabular}{|c|c|c|c|c|c|}
\hline Dependent variable & Parameter & $\begin{array}{l}\text { Standardized } \\
\text { coefficients }\end{array}$ & F-value & $R^{2}(\mathrm{~m})$ & $R^{2}(\mathbf{c})$ \\
\hline \multicolumn{4}{|c|}{ A- Absolute basal area - non-fixing legumes } & 4.23 & 12.37 \\
\hline & Stand age & 0.47 & $27.32^{* *}$ & & \\
\hline & Rainfall & 0.37 & $8.42^{*}$ & & \\
\hline & Stand age $\times$ Rainfall & -0.48 & $13.81^{*}$ & & \\
\hline \multicolumn{4}{|c|}{ B- Relative basal area - non-fixing legumes } & 3.35 & 26.41 \\
\hline & Stand age & 0.39 & $4.18^{\text {n.s. }}$ & & \\
\hline & Rainfall & 0.29 & $1.67^{\text {n.s. }}$ & & \\
\hline & Stand age $\times$ Rainfall & -0.48 & $3.56^{\text {n.s. }}$ & & \\
\hline
\end{tabular}




\section{Supplementary Table 6 | Modelling parameters for the effects of stand age and}

rainfall on legume abundance in Neotropical secondary forests, shown in Fig. 1.

Equations for absolute basal area and relative basal area (RA) of all legume species as well as N-fixing and bipinnate species are shown. Random site intercepts for betweensite variation in initial legume basal area, and random slopes for the variation of the effect of stand age on legume basal area among sites were both included. $N=42$ chronosequence sites.

\begin{tabular}{|c|c|c|}
\hline Dependent variable & $\begin{array}{l}\text { Transformation } \\
\text { on Y }\end{array}$ & Model \\
\hline $\begin{array}{l}\text { Absolute basal area of all } \\
\text { legumes }\end{array}$ & none & $\begin{array}{l}\text { basal area }=-0.15+1.70 \times \text { stand age }-0.18 \times \\
(\text { rainfall } / 1000)+0.08 \times \text { stand age } \times(\text { rainfall } / 1000)\end{array}$ \\
\hline RA of all legumes & $\arcsin$ & $\begin{array}{l}\mathrm{RA}=1.30-0.23 \times \text { stand age }-0.40 \times(\text { rainfall } / 1000) \\
+0.08 \times \text { stand age } \times(\text { rainfall } / 1000)\end{array}$ \\
\hline RA of N-fixing legumes & $\arcsin$ & $\begin{array}{l}\mathrm{RA}=1.34-0.29 \times \text { stand age }-0.43 \times(\text { rainfall } / 1000) \\
+0.10 \times \text { stand age } \times(\text { rainfall } / 1000)\end{array}$ \\
\hline RA of bipinnate legumes & $\arcsin$ & $\begin{array}{l}\mathrm{RA}=0.82-0.13 \times \text { stand age }-0.26 \times(\text { rainfall } / 1000) \\
+0.04 \times \text { stand age } \times(\text { rainfall } / 1000)\end{array}$ \\
\hline
\end{tabular}




\section{References}

63. Mitchell, T. D. \& Jones, P. D. An improved method of constructing a database of monthly climate observations and associated high-resolution grids. Int. J. Climatol. 25, 693-712 (2005).

64. Peña-Claros, M. Changes in forest structure and species composition during secondary forest succession in the Bolivian Amazon. Biotropica. 35, 450-461 (2003).

65. Kennard, D. K. Secondary forest succession in a tropical dry forest: patterns of development across a 50-year chronosequence in lowland Bolivia. J. Trop. Ecol. 18, 53-66 (2002).

66. Piotto, D. "Spatial dynamics of forest recovery after swidden cultivation in the Atlantic forest of southern Bahia, Brazil", Ph.D. thesis, Yale University (2011).

67. Vieira, I. C. G. et al. Classifying successional forests using Landsat spectral properties and ecological characteristics in eastern Amazonia. Remote Sens. Environ. 87, 470-481 (2003).

68. Junqueira, A. B., Shepard, G. H. \& Clement, C. R. Secondary forests on anthropogenic soils conserve agrobiodiversity. Biodivers. Conserv. 19, 19331961 (2010).

69. Madeira, B. G. et al. Changes in tree and liana communities along a successional gradient in a tropical dry forest in south-eastern Brazil. Plant Ecol. 201, 291-304 (2009).

70. Cabral, G. A. L., Sampaio, E. V. S. B. \& de Almeida-Cortez, J. S. Estrutura espacial e biomassa da parte aérea em diferentes estádios successionais de caatinga, em Santa Terezinha, Paraíba. Rev. Bras. Geogr. Fís. 6, 566-574 (2013).

71. Vester, H. F. M. \& Cleef, A. M. Tree architecture and secondary tropical rain forest development - a case study in Araracuara, Colombian Amazonia. Flora 193, 75-97 (1998).

72. Granda Moser, V., Finegan, B., Ramos Bendaña, Z. S., Detlefsen, G. \& Molina, A. Potencial de manejo de bosques restaurados por sucesión natural secundaria en Guanacaste, Costa Rica: composición, diversidad y especies maderables. CATIE Boletín técnico 78, 1-56 (2015).

73. Powers, J. S., Becknell, J. M., Irving, J. \& Perez-Aviles, D. Diversity and structure of regenerating tropical dry forests in Costa Rica: geographic patterns and environmental drivers. For. Ecol. Mgmt. 258, 959-970 (2009).

74. Chazdon, R. L., Brenes, A. R. \& Alvarado, B. V. Effects of climate and stand age on annual tree dynamics in tropical second-growth rain forests. Ecology 86, 1808-1815 (2005).

75. Letcher, S. G. \& Chazdon, R. L. Rapid recovery of biomass, species richness, and species composition in a forest chronosequence in northeastern Costa Rica. Biotropica 41, 608-617 (2009).

76. van Breugel, M., Martínez-Ramos, M. \& Bongers, F. Community dynamics during early secondary succession in Mexican tropical rain forests. J. Trop. Ecol. 22, 663-674 (2006).

77. Mora, F. et al. Testing chronosequences through dynamic approaches: time and site effects on tropical dry forest succession. Biotropica 47, 38-48 (2015). 
78. Orihuela-Belmonte, D. E. et al. Carbon stocks and accumulation rates in tropical secondary forests at the scale of community, landscape and forest type. Agric. Ecosyst. Environ. 171, 72-84 (2013).

79. Miranda Plaza, E. A. "Factores que afectan la estructura de la vegetación en dos paisajes del bosque tropical seco de la Península de Yucatán”, M.S. thesis, Centro de Investigación Científica de Yucatán, México (2014).

80. Dupuy, J. M. et al. Patterns and correlates of tropical dry forest structure and composition in a highly replicated chronosequence in Yucatan, Mexico. Biotropica 44, 151-162 (2012).

81. Lebrija-Trejos, E., Bongers, F., Pérez-García, E. A. \& Meave, J. A. Successional change and resilience of a very dry tropical deciduous forest following shifting agriculture. Biotropica 40, 422-431 (2008).

82. van Breugel, M. et al. Succession of ephemeral secondary forests and their limited role for the conservation of floristic diversity in a human-modified tropical landscape. PLoS ONE 8, e82433 (2013).

83. Denslow, J. S. \& Guzman, S. Variation in stand structure, light and seedling abundance across a tropical moist forest chronosequence, Panama. J. Veg. Sci. 11, 201-212 (2000).

84. Marín-Spiotta, E., Ostertag, E. \& Silver, W. L. Long-term patterns in tropical reforestation: plant community composition and aboveground biomass accumulation. Ecol. Appl. 17, 828-839 (2007).

85. Aide, T. M., Zimmerman, J. K., Pascarella, J. B., Rivera, L. \& Marcano-Vega, H. Forest regeneration in a chronosequence of tropical abandoned pastures: implications for restoration ecology. Restor. Ecol. 8, 328-338 (2000). 